\title{
RECONNAISSANCE OF THE HR 8799 EXOSOLAR SYSTEM. II. ASTROMETRY AND ORBITAL MOTION
}

\author{
L. Pueyo ${ }^{1}$, R. Soummer ${ }^{1}$, J. Hoffmann ${ }^{2}$, R. Oppenheimer ${ }^{3}$, J. R. Graham ${ }^{4}$, N. Zimmerman ${ }^{5,15}$, C. Zhai $^{6}$, J. K. Wallace $^{6}$, \\ F. Vescelus ${ }^{6}$, A. Veicht ${ }^{3}$, G. Vasisht ${ }^{6}$, T. Truong ${ }^{6}$, A. Sivaramakrishnan ${ }^{1}$, M. Shao ${ }^{6}$, L. C. Roberts, Jr. ${ }^{6}$, J. E. Roberts $^{6}$, \\ E. Rice ${ }^{3,7}$, I. R. Parry ${ }^{8}$, R. Nilsson ${ }^{3}$, T. Lockhart ${ }^{6}$, E. R. Ligon ${ }^{6}$, D. King ${ }^{8}$, S. HinkLeY $^{9}$, L. Hillenbrand $^{10}$, D. Hale $^{11}$,

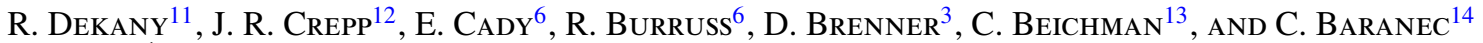 \\ ${ }^{1}$ Space Telescope Science Institute, 3700 San Martin Drive, Baltimore, MD 21218 USA; pueyo@ stsci.edu. \\ A description of the contributions of each author can be found at http://www.amnh.org/project1640 \\ ${ }^{2}$ Department of Physics and Astronomy, Johns Hopkins University, Baltimore, MD, USA \\ ${ }^{3}$ Astrophysics Department, American Museum of Natural History, Central Park West at 79th Street, \\ New York, NY 10024, USA \\ ${ }^{4}$ Berkeley Astronomy Department, 601 Campbell Hall, University of California, Berkeley CA 94720-3411, USA \\ ${ }^{5}$ Department of Mechanical and Aerospace Engineering, Princeton University, Princeton, NJ 08544, USA \\ ${ }^{6}$ Jet Propulsion Laboratory, California Institute of Technology, 4800 Oak Grove Drive, Pasadena, CA 91109, USA \\ ${ }^{7}$ Department of Engineering Science \& Physics, College of Staten Island, 2800 Victory Boulevard, Staten Island, NY 10314, USA \\ ${ }^{8}$ Institute of Astronomy, Cambridge University, Madingley Road, Cambridge CB3 OHA, UK \\ ${ }_{9}^{9}$ Department of Physics and Astronomy, University of Exeter, Physics Building, Stocker Road, Exeter EX4 4QL \\ ${ }^{10}$ Department of Astronomy, California Institute of Technology, 1200 East California Boulevard, MC 249-17, Pasadena, CA 91125, USA \\ ${ }^{11}$ Caltech Optical Observatories, California Institute of Technology, Pasadena, CA 91125, USA \\ ${ }^{12}$ Department of Physics, 225 Nieuwland Science Hall, Notre Dame, IN 46556, USA \\ ${ }^{13}$ NASA Exoplanet Science Institute, California Institute of Technology, Pasadena, CA 91125, USA \\ ${ }^{14}$ Institute for Astronomy, University of Hawai'i at Mānoa, Hilo, HI 96720-2700, USA \\ Received 2014 March 8; accepted 2014 August 4; published 2015 April 10
}

\begin{abstract}
We present an analysis of the orbital motion of the four substellar objects orbiting HR 8799. Our study relies on the published astrometric history of this system augmented with an epoch obtained with the Project 1640 coronagraph with an integral field spectrograph (IFS) installed at the Palomar Hale telescope. We first focus on the intricacies associated with astrometric estimation using the combination of an extreme adaptive optics system (PALM-3000), a coronagraph, and an IFS. We introduce two new algorithms. The first one retrieves the stellar focal plane position when the star is occulted by a coronagraphic stop. The second one yields precise astrometric and spectrophotometric estimates of faint point sources even when they are initially buried in the speckle noise. The second part of our paper is devoted to studying orbital motion in this system. In order to complement the orbital architectures discussed in the literature, we determine an ensemble of likely Keplerian orbits for HR 8799bcde, using a Bayesian analysis with maximally vague priors regarding the overall configuration of the system. Although the astrometric history is currently too scarce to formally rule out coplanarity, HR 8799d appears to be misaligned with respect to the most likely planes of HR $8799 \mathrm{bce}$ orbits. This misalignment is sufficient to question the strictly coplanar assumption made by various authors when identifying a Laplace resonance as a potential architecture. Finally, we establish a high likelihood that HR 8799de have dynamical masses below $13 M_{\text {Jup }}$, using a loose dynamical survival argument based on geometric close encounters. We illustrate how future dynamical analyses will further constrain dynamical masses in the entire system.
\end{abstract}

Key words: astrometry - instrumentation: adaptive optics - instrumentation: spectrographs - methods: data analysis - planetary systems - stars: individual (HR 8799)

\section{INTRODUCTION}

\subsection{Orbital Motion: A Key Element of Direct-imaging Surveys}

High-contrast imaging of nearby stars is a powerful tool to acquire novel insights regarding the architecture and formation history of planetary systems. Such observations are indeed sensitive to substellar companions and faint planets in a separation regime $(\sim>10 \mathrm{AU})$ difficult to reach using indirect methods (Oppenheimer \& Hinkley 2009; Veras et al. 2009; Crepp \& Johnson 2011). They also enable one to survey in the vicinity of young and adolescent stars (McBride et al. 2011; Beichman et al. 2010) and thus provide direct constraints on the early stages of planetary formation and evolution (Spiegel \& Burrows

\footnotetext{
${ }^{15}$ http://www.amnh.org/project1640
}

2011; Baraffe et al. 2010; Brandt et al. 2014). Over the past few years a handful of such objects have been directly imaged (Chauvin et al. 2005; Marois et al. 2008b; Kalas et al. 2008; Lafrenière et al. 2008; Lagrange et al. 2010; Marois et al. 2010b; Ireland et al. 2011). Because their near-infrared radiation is readily available for characterization, their discovery has spurred numerous follow-up photometric (Quanz et al. 2010; Currie et al. 2011, 2012, 2014; Galicher et al. 2011; Janson et al. 2012; Esposito et al. 2013; Skemer et al. 2012) and spectroscopic observations (Janson et al. 2010; Bowler et al. 2010; Barman et al. 2011a, 2011b; Oppenheimer et al. 2013). This wealth of information, only available in favorable configurations in the case of exoplanets detected with indirect methods, has in turn inspired discussions regarding their underlying bulk physical properties and atmospheric chemistry (Madhusudhan et al. 2011; Barman 
et al. 2011a, 2011b; Marley et al. 2012). Their unique loci in the separation-versus-age plane combined with their rich observable astrophysical content make directly imaged exoplanets very compelling comparative exoplanetology objects. Large observational programs, relying on new-generation instruments aimed at identifying more of such faint companions, are currently underway or about to be started (Beuzit et al. 2008; Macintosh et al. 2008; Brandt et al. 2014; Hinkley et al. 2011b). The observations underlying this paper were obtained using one of these instruments: the Project 1640 integral field spectrograph (IFS) (Hinkley et al. 2011b) installed at the Palomar Hale Telescope behind the PALM 3000 adaptive optics system (Dekany et al. 2013).

Using an IFS, one can reveal near-infrared spectroscopic features of substellar companions to nearby stars, study their atmosphere, and infer their bulk physical properties (Barman et al. 2011b, 2011b; Oppenheimer et al. 2013; Konopacky et al. 2013; Hinkley et al. 2013). However, spectroscopic observations cannot fully address uncertainties in the massluminosity relationship of substellar objects at young ages $(<100 \mathrm{Myr})$ because imaging does not directly yield observables commensurate with dynamical masses. They are inferred by folding together estimated age (based on stellar indicators) and mass-luminosity relationships (based on evolutionary models) onto their observed photometric points. Calibrating this relationship at young ages is thus of the utmost importance. A key component of the upcoming large surveys will be to obtain, at least for a subset of the discovered objects, model-independent dynamical mass estimates. For later-type host stars, this can be accomplished by obtaining three-dimensional orbits that combine direct imaging and radial velocity observations (Crepp et al. 2012). For low-mass-ratio binaries with small separations in (AU), this is achieved via direct astrometric monitoring over a full orbital period of the binary pair Dupuy et al. (2009); Konopacky et al. (2010). However, for young sources with orbital periods $>10 \mathrm{yr}$ and a high mass ratio, it is very difficult to observe the gravitational influence of the companion on its host star. One of the most promising avenues for obtaining dynamical masses for such young benchmark objects is to use the companion orbital motion to constrain the second-order dynamical interaction between the various components in a multiple system (Fabrycky \& Murray-Clay 2010) or a single planet and a circumstellar disk (Chauvin et al. 2012; Kalas et al. 2013). In all cases, precise orbital characterization, and thus precise astrometry, is at the crux of this mass determination. Because all future direct-imaging campaigns will rely on an integral field spectrograph as the main survey camera, robust astrometric estimators with such instruments is of critical interest. The first goal of this paper is to introduce such a tool to the high-contrast imaging community. In Section 2 we discuss how to retrieve not only the spectra but also the relative positions of faint planets with respect to their host star, in the regime where they are buried under quasi-static speckles.

\subsection{Orbital Motion in the HR 8799 Multiple System}

HR 8799 is a nearby ( $d \sim 30 \mathrm{pc}$ ) young star (30 Myr), which harbors a multiple planetary system, with four planets orbiting at separations ranging from $\sim 20$ to $\sim 75 \mathrm{AU}$ (Marois et al. 2008b, 2010b). In a parent paper by Oppenheimer et al. (2013), we reported near-infrared $(1-1.8 \mu \mathrm{m})$ spectroscopic observations $(R \sim 40)$ of the four planets in this system. Our results highlighted how the spectral energy distributions of these objects are different from known brown dwarfs and established their spectral diversity, in spite of having formed in the same circum- stellar environment. These spectra are also sensitive to a variety of molecular opacities in the atmosphere of each planet and will thus be the observational foundation of future theoretical work aimed at understanding their complex atmospheric chemistry. The HR 8799 system is extremely interesting from a dynamical mass determination standpoint because of its high multiplicity. Since its discovery, numerous epochs of this system have been reported (Hinz et al. 2010; Esposito et al. 2013; Currie et al. 2011, 2012, 2014; Hinkley et al. 2011a; Galicher et al. 2011; Janson et al. 2010; Lafrenière et al. 2009; Marois et al. 2008b, 2010b; Serabyn et al. 2010; Skemer et al. 2012. These provide a finely sampled orbital coverage starting in 2008. Moreover, Soummer et al. (2011a) recently unraveled the three outermost planets in 1998 HST-NICMOS archival data, yielding a sufficiently large temporal baseline to constrain the eccentricity of the second innermost planet. Before the detection of HR 8799e, various authors considered the dynamical architecture of this system and suggested that the masses of HR $8799 \mathrm{bcd}$ might be lower than estimated in the discovery paper (Marois et al. 2008b) in order for the system to have remained stable over its lifetime (Marois et al. 2010b; Fabrycky \& Murray-Clay 2010). Esposito et al. (2013) recently combined the 1998 HST-NICMOS points with an early estimate for the orbit of HR $8799 \mathrm{e}(\sim 3 \mathrm{yr}$ of temporal baseline) and suggested that indeed the dynamical masses of these planets lie around $\sim 7 M_{\text {Jup }}$.

This paper reports the orbital position of HR 8799 bcde at our P1640 epoch and then establishes the subspace of orbits allowable given the collection of epochs obtained over the past few years. We do so by resorting to a Bayesian analysis using the Markov chain Monte Carlo (MCMC) method. We carry out this work in a effort to complement recent orbit-fitting efforts and dynamical investigations that assumed combinations of coplanarity, mean-motion resonances, or circular orbits. These assumptions were necessary to constrain this degenerate problem to a sufficiently small orbital subspace. The priors in our analysis solely reside in the uncorrelated prior random distribution of each orbital Keplerian element of each planet. We present our results in Section 3 and discuss them in the context of already published work in Section 4. In a subsequent paper, we will fold these constraints on the orbits of each planet into an comprehensive dynamical analysis of this system (A. Veicht et al. 2014, in preparation).

\section{DATA REDUCTION AND METHODS FOR HIGH-CONTRAST ASTROMETRY WITH AN IFS}

\subsection{Observation and Global Instrument Calibration}

HR 8799 was observed with P1640 on 2012 June 14 and 15 under excellent conditions and on 2012 October 5 under median conditions. The June 14 and 15 observations comprised a total of 46 and 31 minutes of exposure time, and 165 minutes of integration time were obtained on 2012 October 5. Details of the observations and conditions and instrumental setup are thoroughly described in Oppenheimer et al. (2013), and we refer readers to that paper for further details. These data took advantage of the interferometric calibration system (Wallace et al. 2009; Pueyo et al. 2010; Vasisht et al. 2014). Because of their high quality and in particular their sensitivity to the two innermost planets, we only consider the June 1415 data in the present paper. When seeking to measure the position of faint companions with respect to their host star, three main sources of uncertainties arise: uncertainties associated with intrinsic instrumental calibrations (distortion, plate scale, 
and absolute north orientation), uncertainties associated with the actual location of the star in the focal plane, and biases induced by the speckle suppression algorithm that is required to disentangle the exoplanetary photons from light scattered by wavefront errors.

We calibrated plate scale and absolute north by observing the visual binary HD 120476 (grade 2 orbit in the Washington Double Star catalog ${ }^{16}$ ) from the Sixth Catalog of Orbits of Visual Binary Stars (Hartkopf et al. 2001) ${ }^{17}$ on the night of June 14. Intrinsic distortions induced by both the PALM 3000 adaptive optics system and P1640 are by design smaller than 0.'1 (Bouchez et al. 2008; Hinkley et al. 2011b)over the small P1640 field of view. Their night-to-night variations have been measured to be of the same order (Zimmerman et al. 2011). We could unfortunately not obtain images of a globular cluster in order to empirically derive a geometric distortion map that is contemporaneous with our HR 8799 observations (Yelda et al. 2010). We mitigate this lack of an empirical distortion reference by nodding the position of our images of HD 120476 over the P1640 detector and deriving a plate scale and P.A. offset at each location. When folding together these measurements, we derived a plate scale of $0 . .01948 \pm 0.00005$ and a rotation of the focal plane array with respect to absolute north of $108^{\circ} .92 \pm 0.5$. Because the host star is hidden by the Apodized Pupil Lyot Coronagraph (Soummer 2005; Soummer et al. 2011b) and the four planets have relative brightness, respectively, of $3.2 \%$, $3.3 \%, 2.9 \%$, and $3.7 \%$ of the mean speckle brightness in their vicinity, the other two uncertainties require particular scrutiny. Below we detail the methods our team developed to address potential systematics and quantify robust confidence intervals for these two sources of uncertainties.

\subsection{Location of the Star in the Focal Plane}

\subsubsection{Relative Alignment and Correction of Atmospheric Differential Refraction}

Our data set is composed images in which the stellar position varies as a result of atmospheric differential refraction (ADR) within a multiwavelength cube and instrument tip-tilt jitter between exposures. Our first step is thus to make sure that this ensemble of point-spread functions (PSFs) is coaligned:

1. Because of the presence of quasi-static speckles, postprocessing is needed to discriminate planets from speckles in these coronagraphic images. Precise subpixel image registration is a necessary condition for the algorithms discussed in Section 2.3 to yield optimal performances.

2. Measuring orbital motion is the primary goal of the present paper. Stellar location in the field of view thus ought to be estimated precisely either in each single slice of each cube or in coadded broadband images composed of slices that have first been aligned in the relative sense.

In this paper we follow the latter approach: we first compensate for ADR and tip-tilt jitter by registering all realizations of the $\mathrm{PSF}$ in the observing sequence to one another. Once relative alignment is achieved, we combined all slices in all cubes in order to estimate stellar position (see Section 2.2.2).

\footnotetext{
16 Note that while there are no reported error bars for the orbital elements of this source, uncertainties in P.A. and separation of this binary can be derived based on contemporaneous observations of this source with Robo AO (Riddle et al., 2014).

17 See http://www.usno.navy.mil/USNO/astrometry/optical-IR-prod/wds/orb for the sixth release of this catalog.
}

We achieve relative registration using cross-correlations between images (Crepp et al. 2011; Pueyo et al. 2012). We found that this method, solely based on the data at hand, did yield better registration on P1640 data when compared to methods based on PSF models. We first start by retrieving the scaling relationship between slices in a cube. Indeed, in an IFS data cube, the PSF of the quasi-static speckle field stretches as the wavelength increases, and this feature can be used to reveal the presence of planets below the noise floor set by wavefront errors (Sparks \& Ford 2002).

However, this scaling relationship prevents us from directly applying cross-correlation-based image-registration algorithms between cubes at different wavelengths. Our first step toward ADR correction thus consists of stretching or squashing all slices to the same scale, usually corresponding to the reference wavelength at the spectral channel of highest throughput in P1640. While this scaling relationship is linear in theory, its behavior as a function of spectral channel can be altered by the Earth's atmosphere or the instrument's dispersion. It is also preferable to retrieve it empirically, based on the data at hand, using a method that is not sensitive to stellar position. This is achieved by correlating the absolute value of the Fourier transform of two PSFs obtained at separate wavelengths. Indeed, the absolute value of the transformed PSF in the $u-v$ plane captures the information relative to the spatial scale of each image and does not depend on the relative centering of the images or stellar location (which is captured by the phase in the $u-v$ plane). Our procedure then goes as follows. The template PSF is transformed using a matrix Fourier transform of scaling unity (equivalent to a fast Fourier transform), and the PSF for which the relative scaling is sought is transformed using a matrix Fourier transform of scaling $\gamma$ (see details in Soummer et al. (2007)). We then proceed to find the value of $\gamma$ that minimizes the cross-correlation of the modulus of the two transformed images. We find that, while the spatial scaling law deviates from the theoretical linear behavior over the full P1640 wavelength range $(0.98-1.75 \mu \mathrm{m})$, it does not vary significantly from exposure to exposure and only needs to be updated on a night-to-night basis, or run to run, depending on observing conditions. Once this scaling law is known, we proceed to either compress or stretch all of the slices in an observing sequence to our chosen reference wavelength.

In a second step, now that all slices are at the same scale, we carry out relative alignment by cross-correlating each slice to a reference image, chosen as the slice at our reference wavelength in the first cube of the observing sequence. We calculate the relative image-alignment offsets of each channel using the subpixel alignment algorithm described in GuizarSicairos et al. (2008). Finally, we stretch or squash all cubes to their natural scale in order to obtain a new series of cubes that have been ADR and tip-tilt corrected. In this set of aligned cubes (in the relative sense) all slices in all cubes of the observing sequence are registered so that their stellar location coincides as well as possible with the stellar location in the reference slices. This corresponds to an empirical correction of both the atmospheric dispersion across each given cube and the tip-tilt jitter between cubes. Note that we compared the result of this relative alignment process with ADR models and found good agreement within $\pm 1 / 10$ th of a pixel for this particular data set and a variety of other P1640 observations (Nilson et al., in preparation).

However, at this stage the absolute stellar location in the focal plane is unknown. This quantity is critical in order to constrain 
the orbital motion of planets around their parent star, and we next show how to estimate it using this set of empirically registered slices.

\subsubsection{Absolute Location of the Star}

Current direct-imaging observations rely on deep images with the host star being saturated in order to identify the planets. The location of the star is estimated using short exposures, combined with the introduction of a neutral-density filter (Marois et al. 2008b, 2010b; Esposito et al. 2013), or using the location of the secondary support structures (Soummer et al. 2011a). In the presence of a coronagraph and a differential tip-tilt closed loop operating to ensure alignment of the star with the focalplane occulting spot (Digby et al. 2006), there is no direct image of the star in the focal plane. This largely complicates the estimation of stellar position. To address this problem, a set of four fiducial stellar PSFs can be introduced in the outer radii of coronagraphic images (Marois et al. 2006b; Sivaramakrishnan \& Oppenheimer 2006; Zimmerman et al. 2010), either using a pupil plane grid associated with the apodizer or by modulating the surface of the deformable mirror. These fiducial "satellite spots" then create an astrometric reference frame that can be used to infer the location of the host star in occulted images. When using an astrometric grid or DM modulation, we can take advantage of the broadband radial elongation of "artificial speckles" to infer the location of the star. The P1640 June observations of HR 8799 were conducted without the pupil plane grid and without DM modulation. Here we demonstrate how the stellar location can still be derived in such images based solely on the radial elongation of "natural speckles" in IFS data. Our method can be applied to configurations for which fiducial astrometric spots are present, and we discuss both cases for the sake of generality. However, it is important to notice that our method relies on the hypothesis that broadband speckles do point toward the stellar location. In general this is not true because ADR, or ADC residuals, does modify the stellar location across the bandpass, and this hypothesis is not rigorously true. However, because in the case of IFS data we reconstruct the broadband image based on narrow band slices that have already been registered to one another, this effect is largely mitigated, whether or not the ADR is corrected in the instrument, and the only residual source of uncertainty corresponds to the stellar motion due to the Earth atmosphere within the narrow bandpass of a P1640 slice. This uncertainty is much smaller than the one arising from the method herein. Thus, for the remainder of the paper we operate under the hypothesis that broadband speckles do point toward the stellar location. For nondispersed data, the chromatic stellar motion ought first to be estimated using ADR, or ADC residuals, models before the method described below can be carried out.

We write a coadded broadband image as $b(x, y)$ where $(x, y)$ denote the focal plane coordinates. Note that the relative alignment between spectral channels is necessary to create the broadband cubes (e.g., we need a cube for which we have established that the star is on the same spaxel, albeit unknown, at each wavelength). In such an image, both speckles and fiducial spots appear as radially elongated structures, which all point toward the stellar position, somewhere behind the focal plane mask. There are two sources of speckle (either "natural" or satellite spot) elongation beyond the characteristic scale of the airy disk: the ADR within each narrowband and low-order wavefront errors. Precisely estimating the location of a speckle in a narrow band slice would require modeling both effects carefully, and we did not pursue this avenue in this paper (this is a complicated exercise that will receive its own scrutiny is an upcoming paper). Instead, we chose a global approach based on speckle elongation across the entire instrument bandpass. Our Radon approach does not estimate the point coordinates to which speckles point at a given wavelength; it measures the point coordinates from which each speckle moves across a full cube of narrow band slices. Using P1640 data with satellite spots, we compared the global approach discussed below with more classical methods based on estimating stellar position based on spots centroid in each slice, and we found good agreement within $\pm 1 / 10$ th pixel.

Our first step consists of using a wavelet filter to emphasize the radial structure that is due to speckles or satellite spots in a broadband image. The cutoff scale of this filter is tuned to the characteristic scale of the structures of interest in the broadband image: a few units of angular resolution when using fiducial spots (whose first and sometimes second airy rings are significantly brighter than the surrounding speckle floor) or a single unit of angular resolution when using natural speckles. This step results in an image in which the radial structures in the broadband image have been emphasized, $b_{F}(x, y)$, as shown in the top two panels of Figures 2 and 3.

In order to illustrate how we derive the position of the star based on the information contained in broadband radial structures, we consider the case of an image composed of a finite number of infinitely thin radial lines of length $L$ all converging onto the point $\left(x_{C}, y_{C}\right)$ :

$$
\begin{aligned}
b_{F}(x, y)= & \Pi\left(\frac{\sqrt{x^{2}+y^{2}}}{L}\right) \\
& \times \sum_{k=1}^{N_{\text {Lines }}} \delta\left(\left(x-x_{C}\right) \cos \alpha_{k}+\left(y-y_{C}\right) \sin \alpha_{k}\right),
\end{aligned}
$$

where $\alpha_{k}$ is the slope angle of each line, $\Pi$ the top-hat function, and $\delta$ the Dirac distribution. In a coordinate system centered at $\left(x_{0}, y_{0}\right)$, the Radon transform of $b_{F}(x, y)$ is given by

$$
\begin{aligned}
R b_{F}(s, \alpha)\left[x_{0}, y_{0}\right]= & \int_{-\infty}^{\infty} b_{F}(t \sin \alpha+s \cos \alpha \\
& \left.+x_{0},-t \cos \alpha+s \sin \alpha+y_{0}\right) d t
\end{aligned}
$$

The Radon transform of the image with the set of radial lines is therefore

$$
R b_{F}(s, \alpha)\left[x_{0}, y_{0}\right]=\sum_{k=1}^{N_{\text {Lines }}} r_{k}(s, \alpha)
$$

where the Radon transform of a single line is the twodimensional image shown in the right panel of Figure 1 and represents the projection of the object along the $s$ vector as a function of the angle $\alpha$. In the case of an image defined as a set of radial lines described by Equation 1 (i.e., similar to a field of radially elongated speckles centered around the star), the Radon transform becomes a superposition of these patterns for a single line at different phase angles. The Radon transform is therefore mostly concentrated along a suite of bright spots corresponding to the angles orthogonal to the radial features in the image. When the center of the coordinate system underlying the Radon transform corresponds to the location of the star, i.e., $\left(x_{0}, y_{0}\right)=\left(x_{C}, y_{C}\right)$, the transform of the ensemble of lines has all bright spots located along the $s=0$ line. Otherwise, 

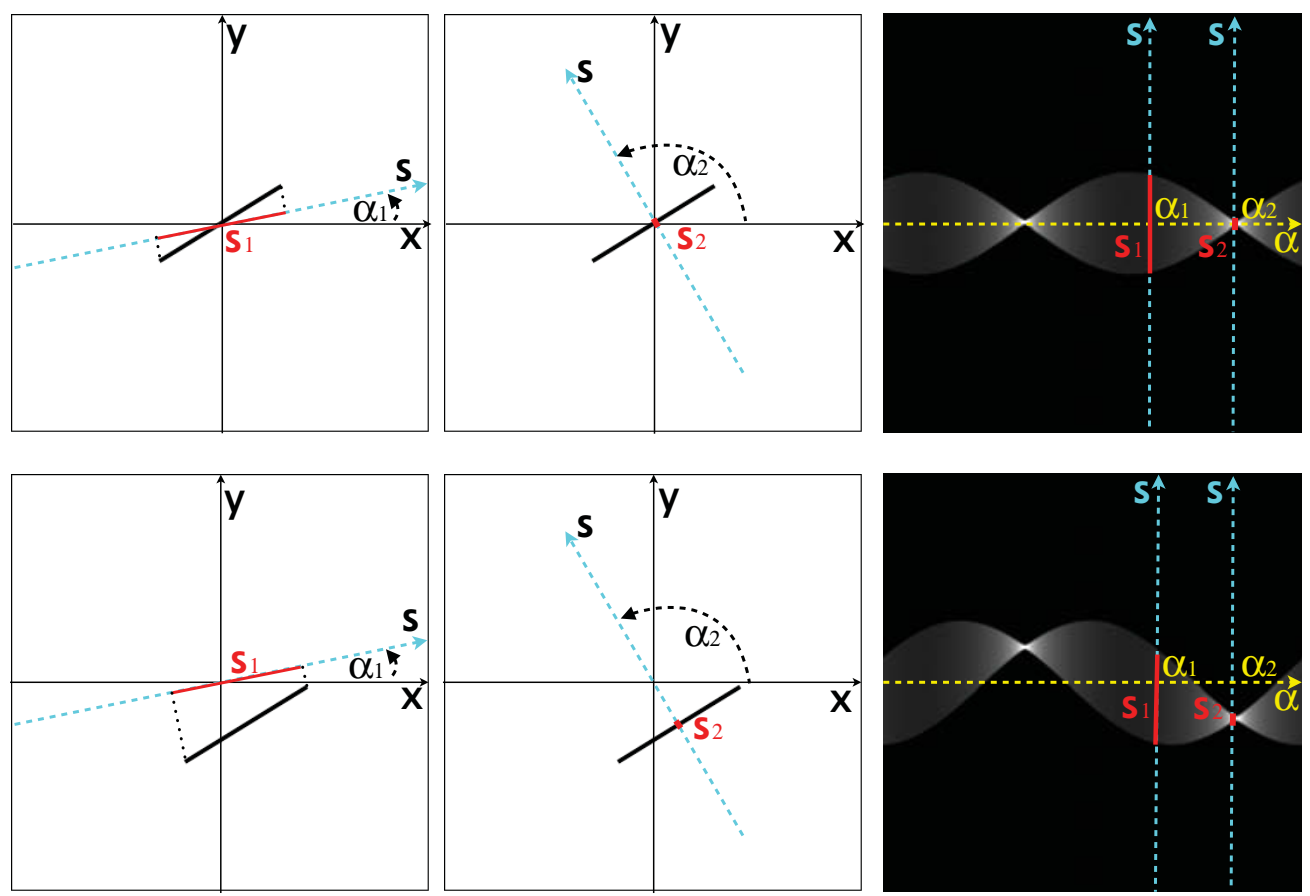

Figure 1. Illustration of the Radon transform of a simple line object (black line) in the case where the line is aligned with both the coordinate center and the Radon transform center $\left(x_{0}, y_{0}\right)=\left(x_{C}, y_{C}\right)$ (top three panels), and in the case where the line is shifted from the Radon transform center (bottom three panels). The Radon transform (right panels) represents the projection along the $s$ vector as a function of the angle $\alpha$. The left figure shows the projection for arbitrary angle $\alpha=\alpha_{1}$ (red line $S_{1}$ ), which can also be seen in the Radon transform at angle $\alpha_{1}$. For the projection angle $\alpha=\alpha_{2}$, the projection is concentrated into a single point in the Radon transform (or a very small spot if the line has a finite thickness). When the line object is aligned with the center of the Radon transform (see top three panels), the bright spots in the Radon transform (at $\alpha=\alpha_{2}+k \pi, k \in \mathbb{Z}$ ) are located at $s=0$. In the case where the line object is offset from the center of the Radon transform, the bright spot for projection angle $\alpha_{2}$ is obtained for $s \neq 0$. This important property is used here to determine the precise location of the star using either the satellite spots or broadband elongated speckles because the Radon transform becomes a superposition of these patterns.

the Radon transform takes the form of a suite of "point-like" bright spots distributed along a trigonometric curve (see, e.g., the bottom three panels of Figure 1). In practice, the radial lines are thick and of finite length (elongated speckles or astrometric spots), but the Radon transform remains very localized in the bright cores, as shown in Figures 2 and 3.

The fact that the Radon transform maps lines onto points is exactly the property needed when seeking to use an ensemble of speckles to estimate the location of the star in a coronagraphic image. We thus estimate the location of the star by calculating the Radon transform of a given broadband image over a grid of purported centers $\left(x_{0}, y_{0}\right)$ and find the location that maximizes the modulus square of the Radon transform over the $s=0$ horizontal axis:

$$
\left(x_{C}, y_{C}\right)=\underset{\left(x_{0}, y_{0}\right)}{\arg \max } \int\left|R b_{F}(0, \alpha)\left[x_{0}, y_{0}\right]\right|^{2} d \alpha .
$$

The bottom panel of Figure 2 shows the contour map of this metric in the case of a generic data cube with fiducial spots. Figure 3 illustrates the particular case useful for this paper, where the star location is solely derived using "natural speckles. In this latter case, because of the noisier nature of the speckles, the constraint on stellar location is less tight than when fiducial spots are present. Moreover, when only using "natural speckles, there exist local maxima outside of this region of interest, as shown in Figure 3, whereas in the case of fiducial spots, the cost function is monotonically decreasing in all directions away from its maximum. However, modern coronagraphs such as P1640 provide absolute tip-tilt telemetry that is precise enough to provide a good first guess for stellar location even in the absence of satellite spots.
Figures 2 and 3 show that the Radon transform of broadband images provides a systematic way to estimate stellar location in IFS broadband images and constrain the uncertainties associated with it. Because upcoming direct-imaging instruments will use an IFS behind a coronagraph for their high-contrast surveys, this method is relevant to all of these projects. More general methods for alignment of such instruments can be found in Savransky et al. (2013), who also emphasized the usefulness of Radon and Hough transforms for high-contrast imaging calibrations and science. The Radon method can be used both to test the instrument (e.g., to calibrate potential noncommon path errors between the differential tip-tilt channel and the final focal plane) and to bolster the astrometric precision of scientific observations. Note that while this method measures the point coordinates from which each speckle moves across a full cube of narrow band slices, residual uncertainties in the stellar locations can arise from speckle elongation within a narrow-band channel (due to ADR within each slice and low-order wavefront errors). These effects broaden the thickness of the radial broadband speckles, which, in turn, transfers some energy from the core of the Radon transform to its wings. As a consequence, the peak of the cost function in Figures 2 and 3 is broadened, thus affecting our uncertainties in stellar position. A comparison of Figures 2 and 3 shows why the introduction of fiducial spots is preferable: they constrain the uncertainty associated with stellar location much more firmly. The uncertainty with fiducial spots is \pm 0.1 pixel, but it is only \pm 0.15 pixels when carried out with natural speckles. However, the ability to constrain stellar location with such an accuracy without fiducial spots demonstrates the advantages of this promising technique. When folding the P1640 plate scale, our analysis of the HR 8799 data 

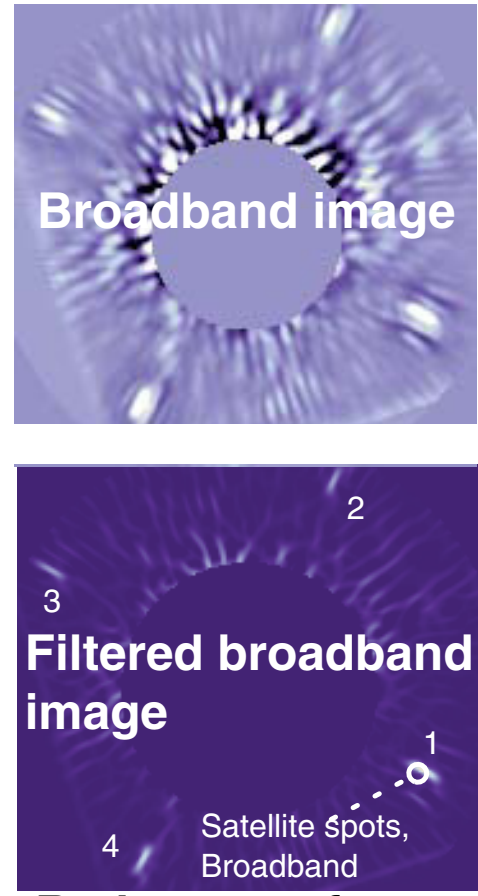

\section{Radon transform}


Figure 2. Determination of stellar location in Radon space with fiducial spots. Top: broadband PSF created by adding aligned hyperspectral slices. Second: broadband PSF propagated through a wavelet filter tuned to the characteristic scale of the four broadband fiducial lines. Third: Radon transform of the second panel, in which the sinusoidal traces of each of four broadband fiducial lines can be identified. When the Radon transform is calculated assuming that the center of the image coincides with stellar location, then the maxima of each sinusoidal trace are located on the $s=0$ axis. Otherwise the scatter of these maxima significantly deviates from this axis. Bottom: cost function calculated by integrating the energy along the $s=0$ axis in Radon space as a function of image centering. The maximum of this quantity lies at the location of the star, and its spread yields an estimate of the uncertainty associated with stellar position.
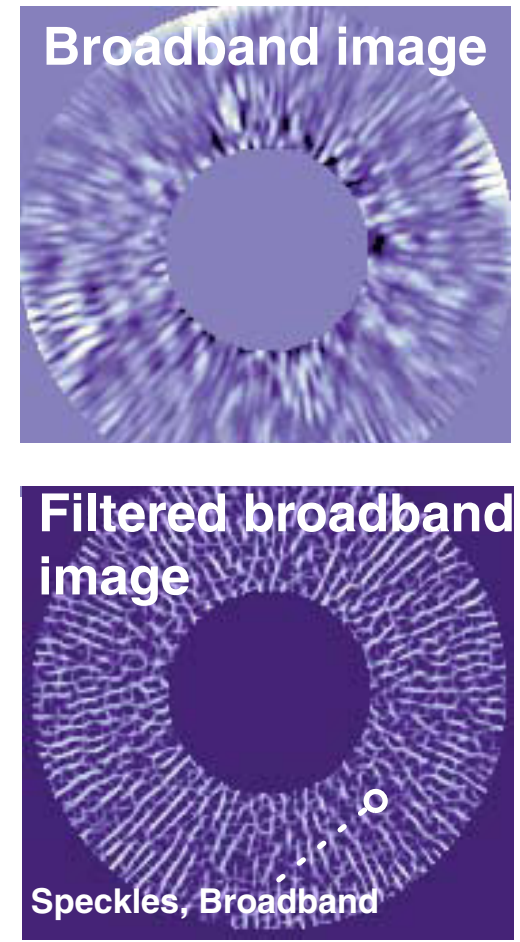

\section{Radon transform}
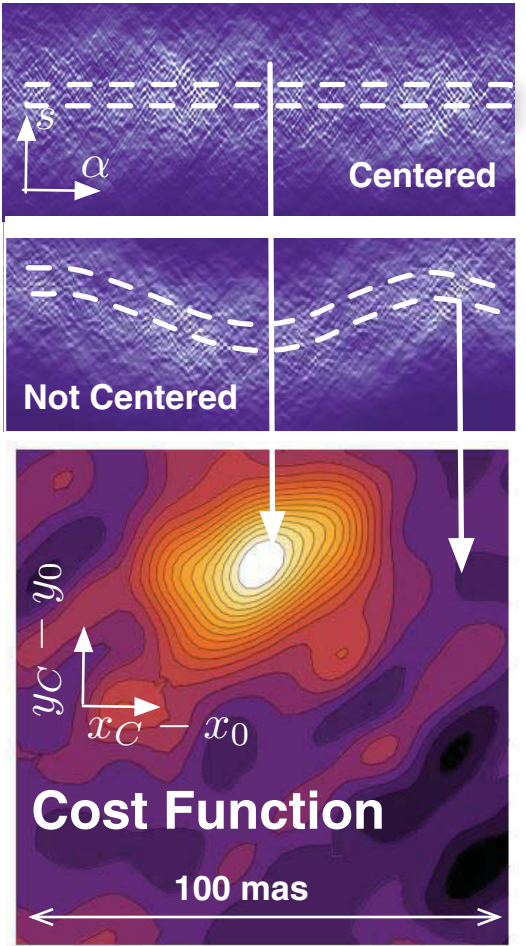

Figure 3. Determination of stellar location in Radon space with "natural speckles." Top: broadband PSF created by adding aligned hyperspectral slices. Second: broadband PSF propagated through a wavelet filter tuned to the characteristic scale of the speckle lines. Third: Radon transform of the second panel, where the sinusoidal traces of the speckles are significantly less pronounced than in the case of fiducial spots. However, when the Radon transform is calculated assuming that the center of the image coincides with the stellar location, then the maxima of most of the sinusoidal trace are still located on the $s=0$ axis. Otherwise the scatter of these maxima significantly deviates from this axis. Bottom: cost function calculated by integrating the energy along the $s=0$ axis in Radon space as a function of image centering. Because "natural speckles" are less pronounced than fiducial spots, the uncertainty associated with stellar position is larger, but it is still well constrained. 
using the "Radon star finder" yields an uncertainty associated with stellar location of \pm 0 '.0033.

\subsection{Planet Locations}

\subsubsection{Context}

In our data, the four planets have a respective relative brightness of $3.2 \%, 3.3 \%, 2.9 \%$, and $3.7 \%$ of the mean speckle brightness in their vicinity, so aggressive post-processing algorithms are required to unravel them in the P1640 data. Contrast limitations due to quasi-static speckles were first discussed in Marois et al. (2006a), and a solution involving image postprocessing was initially introduced by Lafrenière et al. (2007), who devised the locally optimized combination of images algorithm (LOCI). Since then, several variations of this approach have been discussed in the literature, either to improve the contrast (Marois et al. 2010a; Currie et al. 2011; Amara \& Quanz 2012) or to minimize biases on astrophysical observables potentially introduced by the aggressive speckle reduction algorithm. This second problem has received particular scrutiny because thorough characterization of the substellar objects discovered by upcoming direct-imaging campaigns will lead to significant advances in our understanding of exoplanetary systems (Marois et al. 2010a; Pueyo et al. 2012; Soummer et al. 2011a; Brandt et al. 2013; Milli et al. 2012). The proposed solutions rely on two concepts: modifying the least-squares cost function that was introduced in Lafrenière et al. (2007) in order to circumvent degeneracies associated with inverting an ill-posed problem, or calibrating the remaining biases by quantifying the effect of the speckle suppression on synthetic sources. Three recent papers (Soummer et al. 2012; Amara \& Quanz 2012; Fergus et al. 2013) suggested that using principal component analysis (PCA) to analyze direct-imaging data sets can circumvent the problem of inverting a low-rank matrix, and it also provides a framework to rigorously assess how much the astrophysical information is affected by the speckle suppression algorithm. In Oppenheimer et al. (2013) we showed that two of these PCA-based methods can accurately retrieve the spectrum of the four HR 8799 planets in the P1640 data. Because that paper was focused on the interpretation of the spectra, we did not delve into the details of either method or their effect on astrometric estimates. In this section, we describe how to conduct both photometric and astrometric characterizations of faint point sources using the KLIP algorithm (Soummer et al. 2012).

\subsubsection{Nature of Astrophysical Biases}

The algorithms discussed above all rely on using a large collection of PSFs, obtained by using one or several observation strategies (angular differential imaging, spectral differential imaging, reference difference imaging) and subtracting out the quasi-static artifacts in images by fitting them in the leastsquares sense to enhance the detectability of a faint astrophysical signal. This process can lead to two types of biases on the photometric and astrometric estimates of the discovered sources:

1. Fitting bias: where some of the astrophysical source signal is considered as speckle noise by the fitting algorithm (most severe when the inverse problem is ill-posed) and is mistakenly subtracted, even when there is no astrophysical signal present in the reference PSFs.

2. Cross-talk bias: when an astrophysical signal is actually present in the reference PSFs, then self-subtraction can occur, leading to further biases in the information associated with these companions.
We identified these two sources of confusion in Pueyo et al. (2012). In Soummer et al. (2012) we discussed how the fitting bias could be largely mitigated by first transforming the ensemble of reference PSFs into an orthogonal basis set using a Karhunen Loève decomposition. However, our argument relied on the assumption of an ensemble of reference images without any astrophysical signal. While this is true in the case of the HST-NICMOS data discussed in Soummer et al. (2012), it is not generally the case for most observation strategies. As a consequence, cross-talk bias plays an important role in our P1640 data. In Pueyo et al. (2012) we showed how modifying the least-squares cost function and forcing positivity of the fitted coefficients reduces the effect of this bias. Recently, Marois et al. (2014) introduced a promising regularization strategy that is based on modeling astrophysical self-subtraction and including it as a penalty term in the least-squares speckle fitting problem. Here we present an alternative approach that builds upon the decomposition discussed in Soummer et al. (2012). We illustrate its application in the case of point-source detection in integral field spectrograph data, but it can be generalized to any observation strategy and extended objects.

\subsubsection{Reference Libraries for Detection and Characterization}

IFS data is composed of a series of exposures obtained at times $t_{p}$ and at sequential wavelengths $\lambda_{k}$ : we denote such a data set as a collection of images $I_{t_{p}, \lambda_{k}}(x, y)$, with $p \in\left[1, P_{\exp }\right]$ and $k \in\left[1, K_{\Delta \lambda}\right]$, where $P_{\exp }$ is the number of exposures in the observing sequence and $K_{\Delta \lambda}$ the number of spectral channels of the IFS. We assume here that all images have been centered (the relative and absolute centering described above have been carried out). In Crepp et al. (2011) and Pueyo et al. (2012) we showed how the detectability of a faint astrophysical signal could be enhanced in an image using the LOCI algorithm with a PSF library based on rescaled images with the scaling factors resulting from the relative alignment routine. In this paper we use the KLIP algorithm (Soummer et al. 2012) in order to detect point sources in the P1640 data. We set up the least-squares problem associated with KLIP as follows:

1. The image of interest at $\lambda_{k_{0}}$ is partitioned in a series of search zones $\mathcal{S}$ centered on a pixel located at $(x, y)$ of radial extent $\Delta r$ and azimuthal extent $\Delta \theta$.

2. A reference library associated with each search zone is created: for each radial location $r$ in the PSF, only a subset of reference wavelengths $\mathcal{R}_{k_{0}, r, N_{\delta}}$ is kept in the library. This subset is chosen such that $\left|\gamma_{k_{0}, k}-1\right|>N_{\delta} W_{\lambda_{k_{0}}}$ where $W_{\lambda_{k_{0}}}$ is the FWHM of the PSF at $\lambda_{k_{0}}$, and $N_{\delta}$ is a parameter tuned so that it is large enough to avoid self-subtraction (in practice, $N_{\delta} \sim 1-2$ yields detection maps close to the photon noise associated with the speckles in the raw data).

3. The principal components, $Z_{K L}$, of the reference library, $I_{t_{p}, \lambda_{k}}(x, y)$, are calculated and subtracted from the image at $\lambda_{0}$ according to the procedure described in Soummer et al. (2012).

Carrying out this approach over a wide range of parameters $\left(\Delta r, \Delta \theta, N_{\delta}, K_{\mathrm{KLIP}}\right)$ yields deep detection maps, illustrated in Figure 4, where an improvement in contrast of $\sim 30$ reveals the four planets orbiting HR 8799 in the P1640 $H$-band data. Although very practical for detection, this approach breaks down one of the fundamental assumptions in Soummer et al. (2012), the fact that the reference library does not have any astrophysical signal located in the search zone $\mathcal{S}$. Fortunately, this issue can be easily circumvented once a point source has been detected 


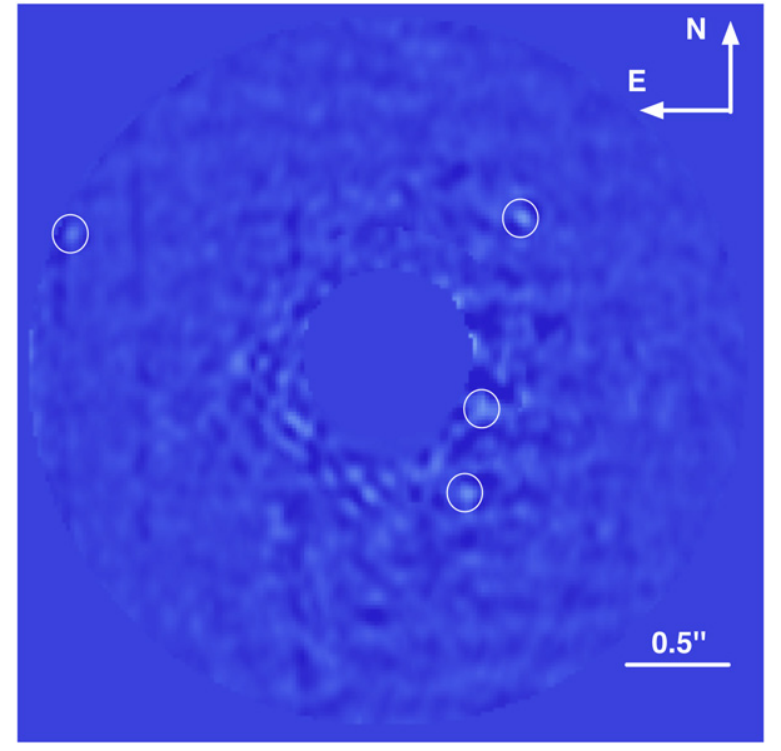

Figure 4. Integrated $H$-band image of the HR 8799 system seen with the P1640 IFS. This image was created by median-combining a series of reductions using the KLIP algorithm over a large set of search-zone geometries, radial exclusion parameters, and principal-component thresholds. While HR 8799 bcd can be detected over a very wide range of parameters, HR 8799e requires a fine-tuning of the azimuthal extent of the search region and the radial exclusion parameter.

at the location $\left(x_{p}, y_{p}\right)$. One can then pose the problem so that there is little astrophysical signal from the companion candidate in the search zone by

1. either choosing characterization zones $\mathcal{C}$ with $r \in\left[r_{p}-\right.$ $\left.\delta r_{C}, r_{p}-\delta r_{C}+\Delta r\right]$ and creating a reference library only with the wavelengths $\lambda_{k} \in \mathcal{R}_{k_{0}, r, N_{\delta}}^{+}$such that $\gamma_{k_{0}, k}-1<$ $-N_{\delta} W_{\lambda_{k_{0}}}$

2. or choosing characterization zones $\mathcal{C}$ with $r \in\left[r_{p}+\delta r_{C}-\right.$ $\left.\Delta r, r_{p}+\delta r_{C}\right]$, and creating a reference library only with the wavelengths $\lambda_{k} \in \mathcal{R}_{k_{0}, r, N_{\delta}}^{-}$such that $\gamma_{k_{0}, k}-1>N_{\delta} W_{\lambda_{k_{0}}}$.

Figure 5 illustrates these two configurations, for which there is no signal from the detected faint companion in the reference library. The variable $\delta r_{C}$ denotes the radial offset between the position of the companion and the edge of the characterization (in the direction of cross talk), and $N_{\delta}$ and $\delta r_{C}$ are the only reduction parameters that can yield cross-talk bias. They ought to be chosen carefully within the bounds $W_{\lambda_{k_{0}}}<\delta r_{C}<$ $N_{\delta} W_{\lambda_{k_{0}}}$. Aside from having to choose yet another reduction parameter, which can actually be done relatively easily as discussed below, the main drawback of this method is that it significantly reduces the number of references available for characterization when compared to the detection algorithm. In the absence of field rotation, we mitigate this effect by choosing strategy (1) described above for blue wavelengths $\lambda_{k_{0}}<\lambda_{K_{\Delta \lambda / 2}}$ and strategy (2) for red wavelengths $\lambda_{k_{0}}>\lambda_{K_{\Delta \lambda / 2}}$. When using all of the cubes from the observing sequence in the reference library, we find that in practice this approach yields levels of speckle suppression comparable to the detection pipeline, except for the few wavelengths near the middle of the spectral bandpass. In the case of P1640, speckle suppression in these few channels is of lesser importance because the middle of P1640's spectral bandpass is located in the atmospheric water band, for which telluric absorption cannot be precisely calibrated given the moderate resolution of the spectrograph. For future instruments such as GPI or SPHERE, this will be mitigated by the ADI observing mode, which will enable

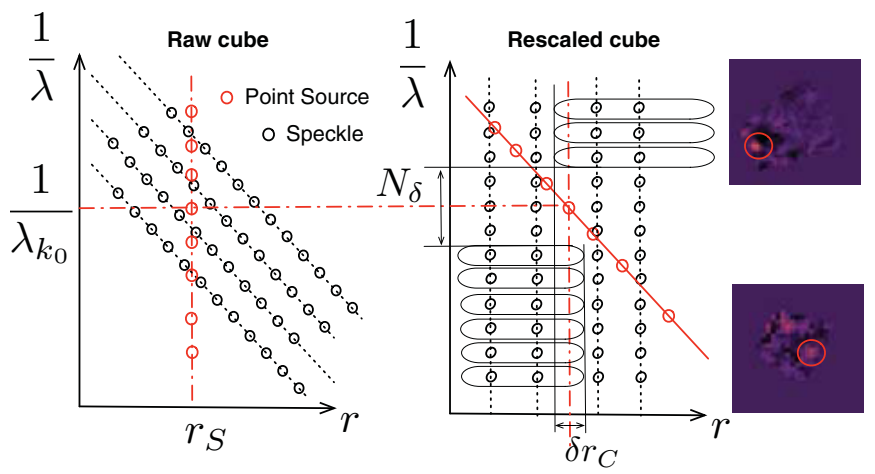

Figure 5. Optimal reference PSF libraries for faint companion characterization when using Integral Field Spectrograph data without angular diversity. Once a companion has been identified and its rough location is known, our goal is to focus on small regions surrounding it and build a library that contains the least amount of the companion's signal possible. To do so, we create a "characterization zone" in which the companion is located near the radial inner (or outer) edge and only use as references rescaled PSFs corresponding to the longer (or shorter) wavelengths. This limits the number of reference images with companion flux present to the few PSFs for which the flux at shorter (or longer) wavelength is still present between the source and the inner (or outer) edge of the characterization zone. This ensures that for each wavelength we are using a PSF library with minimal companion wavelength cross talk. We then vary the parameters $\Delta r$ and $N_{\delta}$ in order to minimize this residual cross talk. Whether we choose the characterization zone so that the companion is near its inner or outer edge is determined by choosing the configuration that will yield the largest "companion-free" PSF library.

combining the radial offset of characterization $\mathcal{C}$ zones described above with an azimuthal offset, thus significantly enlarging the characterization "companion-free" reference library.

\subsubsection{Forward Modeling: Principles}

Once an adequate PSF library has been set up with a minimal amount of companion signal in the reference PSF characterization zone, the forward-modeling approach suggested in Soummer et al. (2012) can be carried out. For simplicity we use the notations in Soummer et al. (2012): the target image at wavelength $\lambda_{0}$ and exposure $t_{0}$ is written as $T(x, y)=I_{t_{0}, \lambda_{k}}(x, y)$, and the ensemble of references $R_{q}(x, y)=I_{t_{p}, \lambda_{k}}(x, y)$ with $\lambda_{k} \in \mathcal{R}_{k_{0}, r, N_{\delta}}, \quad p \in\left[1, P_{\exp }\right]$. The set of principal components of this library is $Z_{q}^{K L}(x, y)$. The reduced image is then

$$
F(x, y)=T(x, y)-\sum_{q=1}^{K_{\text {klip }}}\left\langle T, Z_{q}^{K L}\right\rangle_{\mathcal{C}} Z_{q}^{K L}(x, y) .
$$

We assume a known model $S(x, y)$ of a point source PSF at wavelength $\lambda_{k_{0}}$ with a normalized flux. Under the assumption that the noise in the reduced image $F(x, y)$ is Gaussian and of zero mean, a least-squares estimator yields unbiased values for the brightness and location of a point source. In order to accommodate for the fact that this assumption might not be true over the entire characterization zone $\mathcal{C}$, we can write this cost function over a fitting region $\mathcal{F} \in \mathcal{C}$. We find that for our HR 8799 P1640 data, a fitting zone that spans the entire characterization zone yields unbiased single-channel astrometry and photometry (Figure 6). However, this might not be the case for other instruments or for fainter sources in P1640 data. In the general case of distinct fitting and characterization zones, we solve for the location of the point source $\left(\tilde{x}_{s}, \tilde{y}_{s}\right)$ and its flux $\tilde{f}_{s}$ by minimizing the least forward modeling cost function 


\section{Single wavelength baises at $0.5 "$}
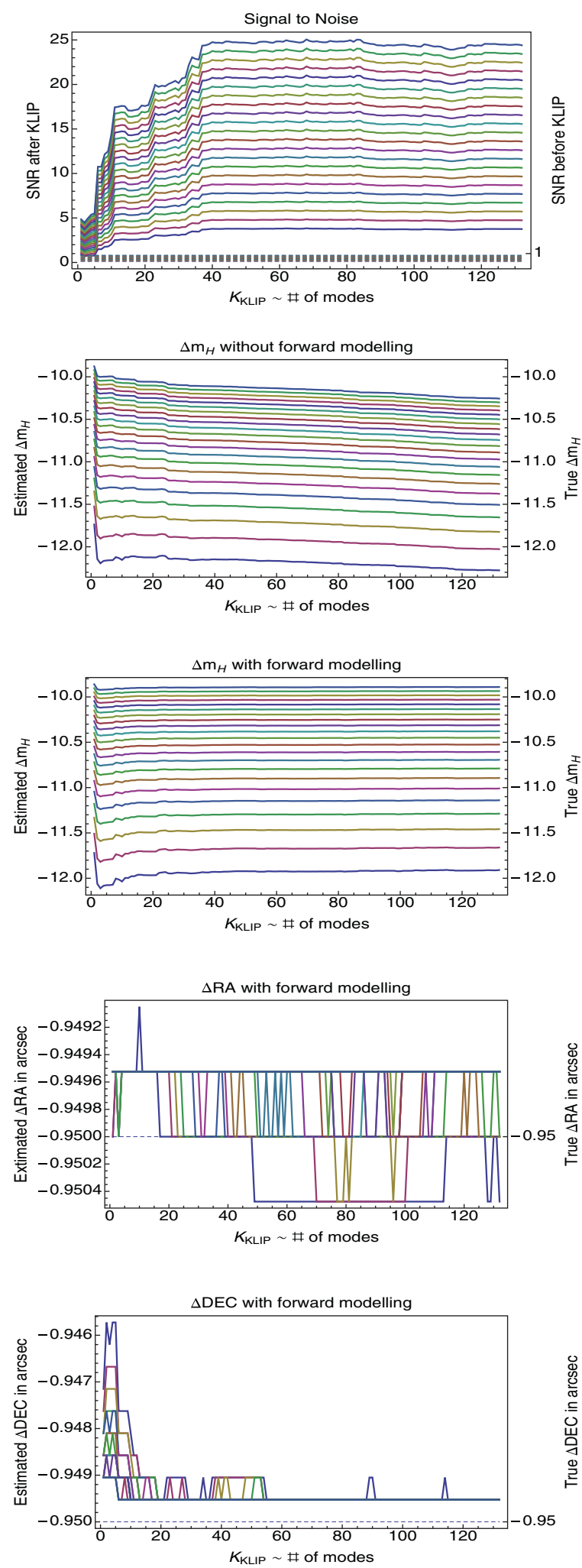

Single wavelength baises at 1"


Figure 6. Left: single-wavelength photometric and astrometric biases at 0.5 . We injected a series of synthetic point sources of various brightness in the $1.3 \mu \mathrm{m}$ P1640 spectral channel. For this test we did not inject any companion flux in other wavelength slices of the IFS data cube: this represents the ideal of a reference cube that is $100 \%$ companion free. We consider the case of the fitting zone $\mathcal{F}$ that overlaps with the characterization zone $\mathcal{C}$. Without forward modeling, the photometry of the reduced data is underestimated because of self-subtraction: as the number of modes increases, the $\mathrm{S} / \mathrm{N}$ of the point source increases, but its estimated flux becomes more and more biased. On the other hand, photometry is preserved in using the forward modeling to take into account this algorithmic flux depletion. Moreover, once a sufficient number of modes has been taken into account, the forward modeling yields very precise estimates of the synthetic source position in the focal plane. Right: single-wavelength photometric and astrometric biases at $1^{\prime \prime}$ : same exercise as on the left except that now the synthetic point source is detected with a higher statistical significance. Note that the spectrophotometric and astrometric biases are further reduced in this case. 
described in Soummer et al. (2012):

$$
\begin{aligned}
& \left(\tilde{x}_{s}, \tilde{y}_{s}, \tilde{f}_{s}\right)=\underset{\left(x_{s}, y_{s}, f_{s}\right)}{\arg \min } \\
& \quad \times\left\|F-f_{S}\left(S-\sum_{q=1}^{K_{\text {klip }}}\left\langle S\left(\cdot-x_{S}, \cdot-y_{S}\right), Z_{q}^{K L}\right\rangle_{\mathcal{C}} Z_{q}^{K L}\right)\right\|_{\mathcal{F}}^{2},
\end{aligned}
$$

where the - represents the dummy integration variable for the inner product. Because this is a quadratic cost function, the point-source coordinates can be estimated using a matched filter approach, as the location of the maximum of the cross correlation between the reduced image and the PSF model propagated though the PCA filter:

$$
\left(\tilde{x}_{s}, \tilde{y}_{s}\right)=\underset{\left(x_{s}, y_{s}\right)}{\arg \max }\left\{C_{\left(x_{s}, y_{s}\right)}\left(F, S, K_{\mathrm{klip}}\right)\right\}
$$

with

$$
\begin{gathered}
C_{\left(x_{s}, y_{s}\right)}\left(F, S, K_{\text {klip }}\right)=\left\langle F, S\left(x-x_{S}, y-y_{S}\right)\right\rangle_{\mathcal{F}} \\
-\sum_{q=1}^{K_{\text {klip }}}\left\langle S\left(x-x_{S}, y-y_{S}\right), Z_{q}^{K L}\right\rangle_{\mathcal{C}}\left\langle F, Z_{q}^{K L}\right\rangle_{\mathcal{F}},
\end{gathered}
$$

where we have indicated the integration variables $x$ and $y$ in the terms where the explicit search variables $x_{S}$ and $y_{S}$ appear. Once the true location of the point source $\left(\tilde{x}_{s}, \tilde{y}_{s}\right)$ has been estimated, its flux is then given by the ratio of the reduced image-model PSF correlation and the estimate of the flux loss due to the fitting bias:

$$
f_{s}=\frac{C_{\left(\tilde{x}_{s}, \tilde{y}_{s}\right)}\left(F, S, K_{\mathrm{klip}}\right)}{\left\|S-\sum_{q=1}^{K_{\mathrm{klip}}}\left\langle S\left(\tilde{x}_{s}-x_{S}, \tilde{y}_{s}-y_{S}\right), Z_{q}^{K L}\right\rangle_{\mathcal{C}} Z_{q}^{K L}\right\|_{\mathcal{F}}^{2}} .
$$

Equations (7)-(9) describe how to take advantage of the KLIP algorithm to derive the focal plane location and the brightness of a faint point source hidden under speckles in high-contrast imaging data. The major conditions for these equations to be valid (and yield astrophysical estimates that are unbiased) are that no companion signal is present in the portion of the image used for characterization, and that the residual noise in the fitting zone is Gaussian of zero mean. We earlier showed how to build a PSF library using IFS data, which almost satisfies the former. The ability to reach Gaussian noise after ADI subtractions was demonstrated by Marois et al. (2008a); this result remains true for LOCI- or KLIP-based subtractions and can be achieved by choosing adequate geometries for characterization zones and tuning $K_{\mathrm{KLip}}$ (e.g., using a thorough parameter search to minimize residual speckle noise as in Soummer et al. (2011a)). In the following section, we test the accuracy of the approach described by Equations (7) to (9) using a synthetic data set that strictly satisfies this condition. Then, in Section 3 we illustrate how to practically derive astrophysical observables and their uncertainties using our HR 8799 data set, for which the "companion-free PSF library" condition is not strictly true. In particular we discuss how to mitigate the residual biases that are introduced in this realistic case.

\subsubsection{Forward Modeling: Results}

Figure 6 illustrates the result of this approach on a synthetic companion injected into the P1640 data. The purpose of these simulations is to assess the accuracy of the forward modeling under the "companion-free PSF library" assumption, and our test data are thus built as such (e.g., using a synthetic companion that is only present in one spectral channel). The findings of this numerical experiment can be summarized as follows:

1. Photometric bias: In our test cases, forward modeling with KLIP circumvents the algorithmic flux depletion due to the fitting companion's flux with speckles discussed in Pueyo et al. (2012). It yields photometric estimates with a bias smaller than 0.02 magnitudes.

2. Photometric uncertainty: For a source with signal-to-noise ratio $(\mathrm{S} / \mathrm{N})$ of $\sim 3$, the photometric uncertainty due to the algorithm (photometric scatter across the number of modes $K_{\text {KLIP }}$ after forward modeling) is $\sim 0.05 \mathrm{mag}$. For sources with $\mathrm{S} / \mathrm{N} \sim 20$, the photometric uncertainty due to the algorithm is $<0.01$ magnitudes. Note that these numbers correspond to the part of the parameter space for which the point-source detectability is somewhat independent of the number of modes used in the PSF subtraction $\left(K_{\mathrm{KLIP}}>40\right)$.

3. Astrometric bias: In our test cases, forward modeling with KLIP yields an astrometric estimate of a bias smaller than 0 .'0005, corresponding to $\sim 1 / 40$ pixel.

4. Astrometric uncertainty: Once the number of modes is sufficient, then the astrometric uncertainty due to the algorithm (astrometric scatter across the number of modes after forward modeling) is 0 .'001 for sources with $\mathrm{S} / \mathrm{N} \sim 3$ and 0 . $0005(\sim 1 / 40$ pixel) for sources with $\mathrm{S} / \mathrm{N} \sim 20$.

Note that these results were obtained when using a fitting region $\mathcal{F}$ that is exactly the size of the characterization zone $\mathcal{C}$ : this choice was driven by the fact that the residual noise in the characterization zone chosen for our test data was indeed Gaussian of zero mean. When this is not the case, then one can either use a subset of the characterization region as the fitting region or simply change the geometry of $\mathcal{C}$ in an attempt to improve the residual-noise statistics. We tested both approaches and did not observe any fundamental differences, and we decided that in practice we would solely use the second approach in our P1640 pipeline for the sake of simplicity.

Naturally, when using real data, the ideal performances above will be severely affected when the various assumptions underlying the simulations in Figure 6 do break down. Namely, the PSF model used for the forward modeling will not strictly be equal to the actual companion's PSF, the residual speckles after KLIP might not be Gaussian of zero mean, and the PSF library will not be completely "companion free. In particular, the absence of field rotation of P1640 results in PSF libraries that are solely based on wavelength diversity. The P1640 chromatic lever arm is actually not large enough to ensure that the "companionfree references" condition is strictly enforced, in spite of our careful selection of the geometries and PSF libraries described in Figure 5. We present in Section 3 our methodology to derive spectrophotometric and astrometric estimates even when these assumptions are only loosely met and illustrate our methodology in the case of HR 8799bcde.

\section{ASTROMETRY AND ORBITAL MOTION OF HR 8799BCDE}

\subsection{Position of Planet in Detector Coordinates}

In a recent paper, Soummer et al. (2011a) illustrated using HST-NICMOS data how overly aggressive PSF subtraction could substantially bias astrometric estimates. We showed in 
Figure 6 that in the case of a companion-free PSF library and a reduced image with zero mean Gaussian residual noise, astrometric biases were mitigated when using the KLIP algorithm. In reality, in spite of all of the efforts described above, there is no guarantee that these assumptions will strictly hold. We thus resort to exploring the algorithmic parameter space in order to identify the configurations for which these assumptions are met as well as possible. Our estimates for spectrophotometry and astrometry of the faint source correspond to the average of these observables over the ensemble of "well-behaved" reductions. Note, however, that this is not the same "blind" parameter search described in Soummer et al. (2011a) because we have identified the main assumptions whose validity we are seeking to test for a given data set. We thus vary

1. the geometry of the characterization zone $\mathcal{C}$ in order to test the residual speckle statistics (e.g., whether or not they are Gaussian of zero mean). Note that the size of the fitting region $\mathcal{F}$ could also be varied as well, but we find that, for P1640 data, changing the size of the geometry of the characterization zone is sufficient.

2. the parameters $\left(N_{\delta}, \delta r_{C}\right)$, in order to test the validity of the "companion-free" assumption. Note that varying $N_{\delta}$ also affects the efficiency of the speckle suppression (and thus the statistics of the residual speckles) because having more correlated PSFs (e.g., corresponding to a wavelength as close as possible to science wavelength) generally yields less noisy postsubtraction residuals.

3. the number of principal components utilized for the subtraction, $K_{\mathrm{KLIP}}$, which will affect the statistics of the residual speckles and also provide a very insightful diagnostics tool.

In general, for a given characterization zone geometry and given $\left(N_{\delta}, \delta r_{C}\right)$, the behavior of spectrophotometry and astrometry as a function of $K_{\mathrm{KLIP}}$ can be divided into three regimes:

1. When $K_{\mathrm{KLIP}}$ is small, the estimated companion's location varies both across wavelengths and values of $K_{\mathrm{KLIP}}$, which means that the PSF subtraction is not aggressive enough and residual speckles are biasing the astrometric estimate.

2. When $K_{\mathrm{KLIP}}$ is large, then the companion flux substantially changes with $K_{\mathrm{KLIP}}$. This means that some of the companion's flux in nearby spectral channels of interest is actually included in the principal components. This occurs because the PSF library is not completely "companion free. Although the small contribution of the companion to the PSF library is not captured by the first modes of the Karhunen-Loève decomposition, its influence on the estimated spectrum starts to become more prominent when $K_{\mathrm{KLIP}}$ is large enough.

3. When $K_{\mathrm{KLIP}}$ is in an intermediate regime, then neither the astrometry nor the spectrophotometry vary with $K_{\mathrm{KLIP}}$ : this is the part of the parameter space that is useful to infer astrophysical estimates.

The boundaries between these regimes is a function of the geometry of the characterization zone, of $\left(N_{\delta}, \delta r_{C}\right)$, and of course of the statistical significance of the companion's detection. We thus execute a parameter space search over 16 different combinations of $\left(N_{\delta}, \delta r_{C}, \Delta r, \Delta \theta\right)$ near the detected location of the companion and vary the number of eigenmodes in the PSF subtraction from one to 130 . This parameter search results in $\sim 2000$ spectra and detector coordinates for each one of the HR 8799 planets. From these $\sim 2000$ spectra, we discard any values that are either in regime (1) for which the astrometric position of the companion is not consistent between wavelength channels or in (2) in which a sharp flux drop is detected with a small change in the number of modes. Finally, we further trim the subset (3) by only keeping the spectra associated with images that exhibit a local $\mathrm{S} / \mathrm{N}>3$. Note that for this paper focused on astrometry, we only consider the $H$-band data because P1640 detections in that bandpass present the highest statistical significance for HR 8799bcde.

Note that part of this parameter search could be alleviated by using the t-LOCI technique developed by Marois et al. (2014), which add a penalty term to the least-squares inversion problem to take into account the predicted location of a companion's flux in the reference images. However, this requires the use of a template spectrum in order to operate optimally: although it presents a substantial gain by reducing the algorithmic parameter search, it is hampered by the need to test a suite of hypotheses regarding the nature of the atmosphere of the astrophysical source. Here we chose to limit ourselves to methods that do not make any assumptions on the properties of the detected companion and discuss the trade-offs associated with spectrophotometry and astrometry in IFS data. While the boundaries between the regimes (1), (2), and (3) can seem ad hoc, the transition between each regime can be easily quantified, and the above procedure can be automated. We have not done so in the early stages of Project 1640 in order to gain insights regarding the synergy between various observing-scenario PCA-based reduction methods. Our team has now successfully extracted spectra and astrometry from a handful of faint sources four orders of magnitude fainter than their host star (Oppenheimer et al. 2013; Hinkley et al. 2013), and we will soon proceed to an automation of this process.

Figure 7 shows a sample of the ensemble of $H$-band spectra and astrometry for HR $8799 \mathrm{bc}$ that belongs to the regime (3) of "well-behaved" astrophysical observables. Because they are detected at $\mathrm{S} / \mathrm{N}>10$, the residual spectrophotometric scatter in this regime is on the order of $10 \%$, which is more accurate than the precision reported by the d-LOCI algorithm at these levels of contrast Pueyo et al. (2012). Moreover, for the channels with the most signal, the astrometric scatter is below 0.2 pixel $\left(\sim 0^{\prime}\right.$.004 for P1640), which is quite remarkable given the flux ratios between planets and raw speckles. We conservatively derive our "detector-based" astrometric error bars as the standard deviation of all estimates in regime (3) over all wavelengths in the shaded regions. The spectrophotometric error bars in Hinkley et al. (2013) and Oppenheimer et al. (2013) are also derived as the standard deviation of all of the spectra in ensemble (3). The case of HR 8799de illustrates how to estimate uncertainties when the astrometric scatter becomes larger due to lesser signal to noise in the detection. Figure 8 displays the ensemble (3) for the two inner planets in the HR 8799 system: the variation of astrophysical observables across wavelength and $\left(N_{\delta}, \delta r_{C}, \Delta r, \Delta \theta, K_{\mathrm{KLIP}}\right)$ is significantly larger in this "wellbehaved regime" than in the case of HR $8799 \mathrm{bc}$. This can be easily explained by the necessity to use small values of $N_{\delta}$ in order to detect these objects in our P1640 data, which yields a reference library that is more contaminated by residual planet flux at adjacent wavelength. As a final sanity check, we overlay on top of the astrometric estimates the radial trace of a putative residual speckle at this location of the focal plane. Using this information, we can establish that these are the only point sources in their respective neighborhoods of the focal plane whose astrometric signature as a function of wavelength does not follow the radial trace of a residual speckle. We derive 


\section{HR8799b}
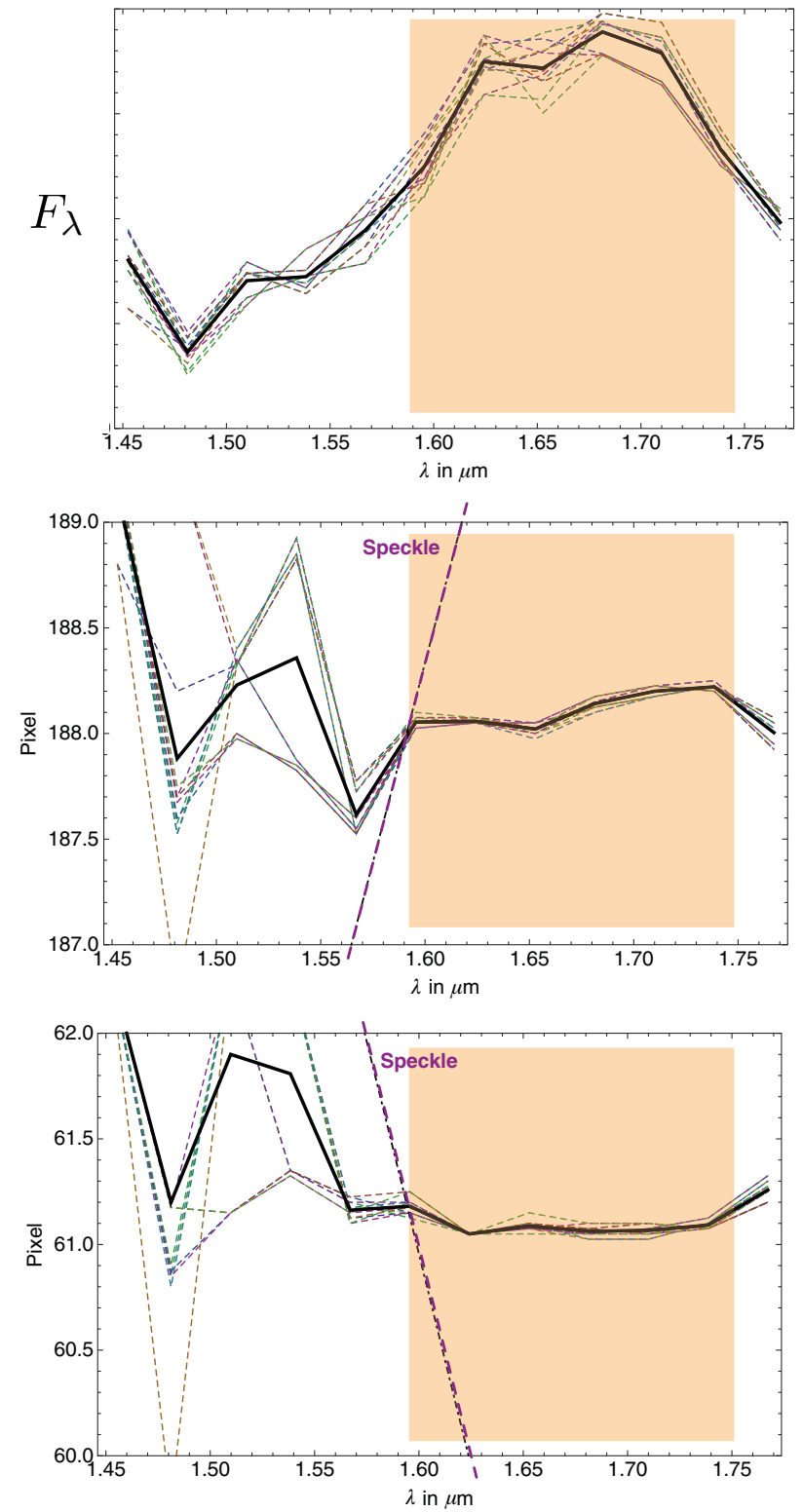

HR8799c
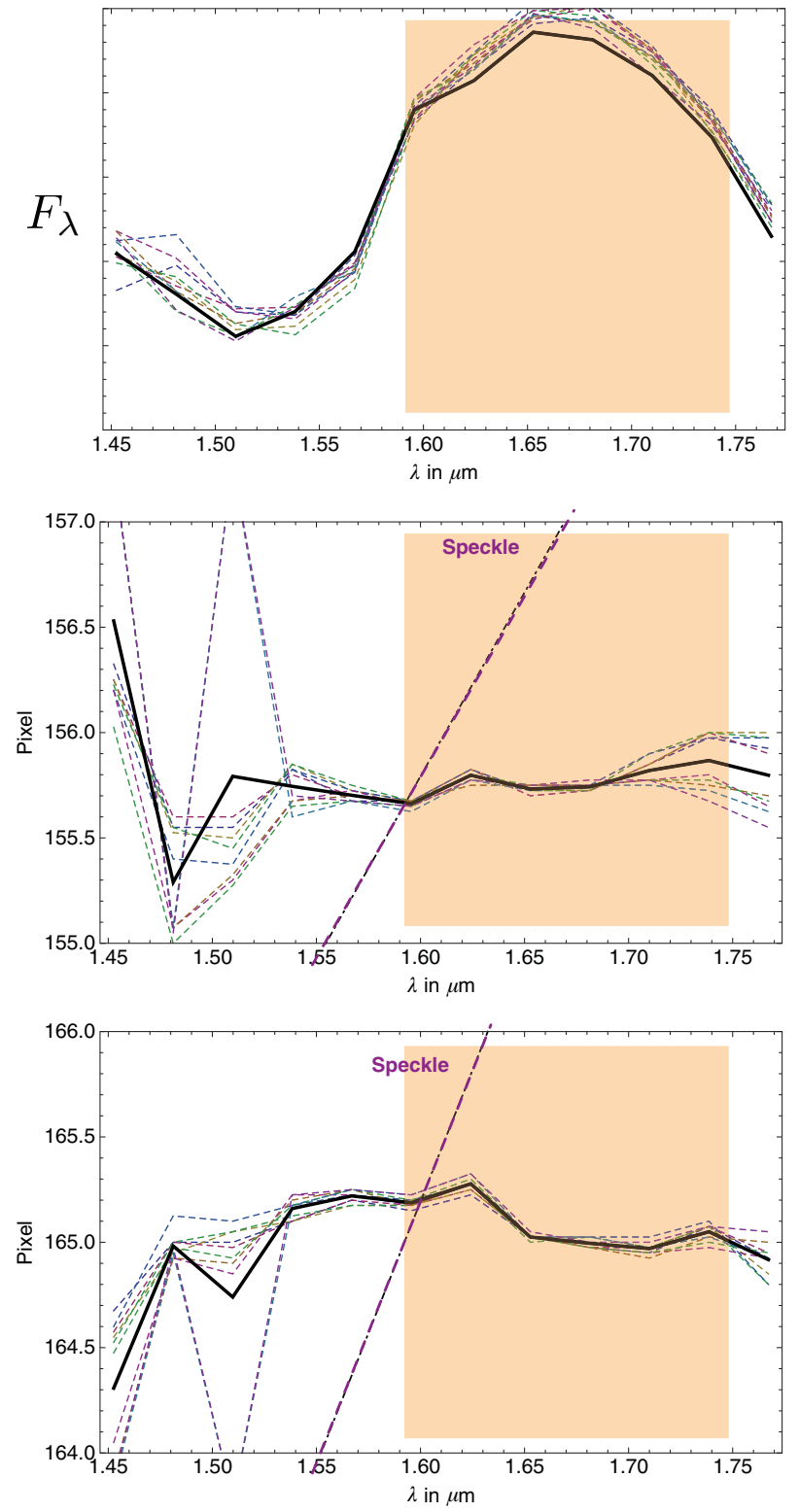

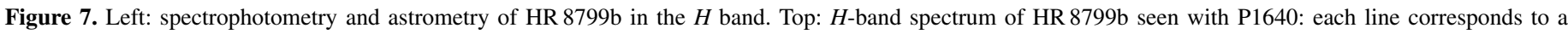





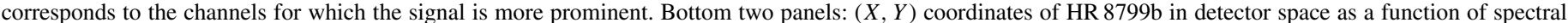

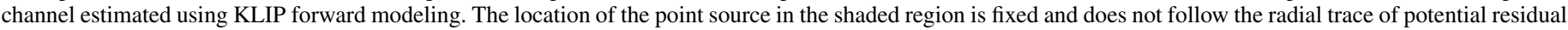
speckle at this location (dashed dot line). Right: spectrophotometry and astrometry of HR 8799c in the $H$ band: same as left panel in the case of HR 8799c.

error bars on the spectrophotometry and astrometry in a similar fashion as we did for HR $8799 \mathrm{bc}$.

\subsection{Astrometry}

Armed with the planets' location and detector coordinates, the stellar location in the focal plane, the instrument's plate scale, and the position angle of absolute north, we can estimate the position of the HR8799 planets relative to their host star in our epoch of observations. The uncertainties associated with the relative astrometry of our epoch are then derived as the root mean squared of the uncertainties associated with each one of these three quantities. Our astrometric estimates and the error
Table 1

HR 8799bcde Relative Astrometry, 2012 June

\begin{tabular}{|c|c|c|c|}
\hline Planet & Epoch & $\Delta$ R.A. & $\delta$ Decl. \\
\hline HR 8799b & 2012.4481 & $1^{\prime \prime} .563 \pm 0^{\prime \prime} .005$ & $0.706 \pm 0.005$ \\
\hline HR 8799c & 2012.4481 & $0^{\prime \prime} .558 \pm 0^{\prime \prime} .004$ & $-0.765 \pm 0 . \prime 004$ \\
\hline HR 8799d & 2012.4481 & $0^{\prime \prime} 323 \pm 0^{\prime \prime} .006$ & $0^{\prime \prime} .529 \pm 0.006$ \\
\hline HR 8799e & 2012.4481 & $0^{\prime \prime} 366 \pm 0^{\prime \prime} .006$ & $0.090 \pm 0.006$ \\
\hline
\end{tabular}

budget associated with them are shown respectively in Tables 1 and 2. For HR 8799bc, the largest uncertainty stems from our limited knowledge of stellar location in the focal plane (because we are deriving this quantity based on elongation of natural 

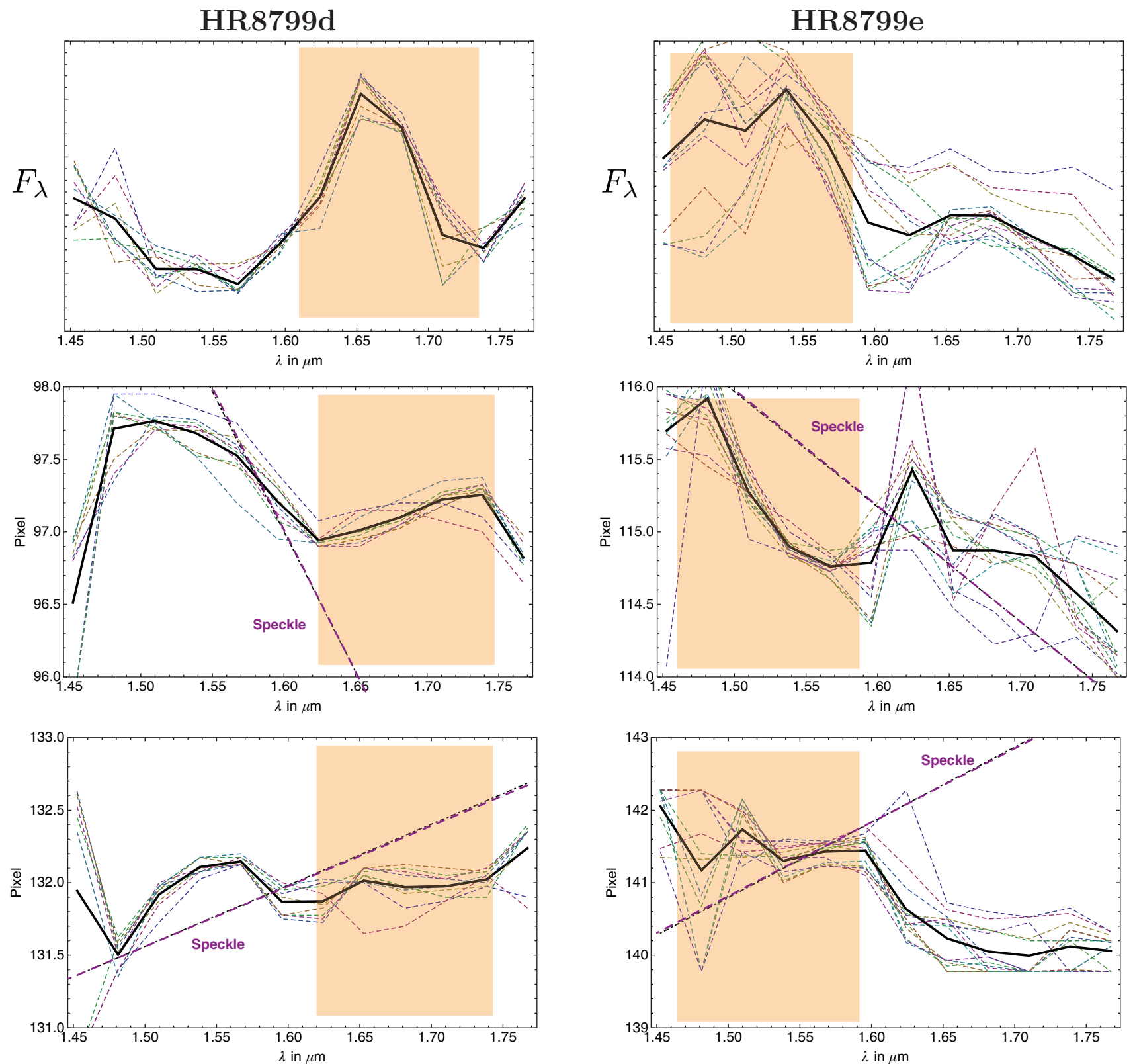

Figure 8. Left: spectrophotometry and astrometry of HR 8799d in the $H$ band. Top: $H$-band spectrum of HR 8799d seen with P1640: each line corresponds to a given data reduction with fixed zone geometry, exclusion parameters, and $K_{\mathrm{KLIP}}$. These spectra were selected according to the criteria described in the text and are anchored in the regime for which the astrophysical estimates cannot be biased either by residual speckles or by overly aggressive PSF subtraction. The shaded band corresponds to the channels for which the signal is more prominent. Bottom two panels: $(X, Y)$ coordinates of HR $8799 \mathrm{~b}$ in detector space as a function of spectral channel estimated using KLIP forward modeling. Because the statistical significance of the detection is lesser than in the case of HR $8799 \mathrm{bc}$, the scatter on the location of HR 8799d, in the shaded region, has increased. However, its overall trend does not follow the radial trace of potential residual speckles at this location (dashed dot line), which ensures that we are indeed detecting an actual astrophysical source, albeit with a larger uncertainty in its astrometric location. Right: spectrophotometry and astrometry of HR 8799e in the $H$ band: same as left panel in the case of HR 8799e. The detection is now close to marginal, and the uncertainty in the source location becomes quite large. However, it is the only point source in this area of the focal plane whose astrometric signature as a function of wavelength does not follow the radial trace of a residual speckle.

speckles instead of using satellite spots). For HR 8799de, the largest uncertainty stems from the aggressiveness of the speckle suppression that is necessary for a statistically significant detection. Figure 9 illustrates the published relative astrometry epochs in conjunction with our P1640 epoch. The most likely orbits resulting from our orbital motion analysis, discussed next, are overlaid on these points with a different color for each planet. We will keep this color-coding through the remainder of the paper when comparing the respective orbital elements of each planet to one another and constraining the orbital architecture of this system.

\subsection{Orbital Motion}

Because HR 8799 is the only directly imaged multiple planetary system to this day, the orbital motion of the four planets has been widely discussed in the literature. Published analyses of the orbital architecture of this system can be divided into two 

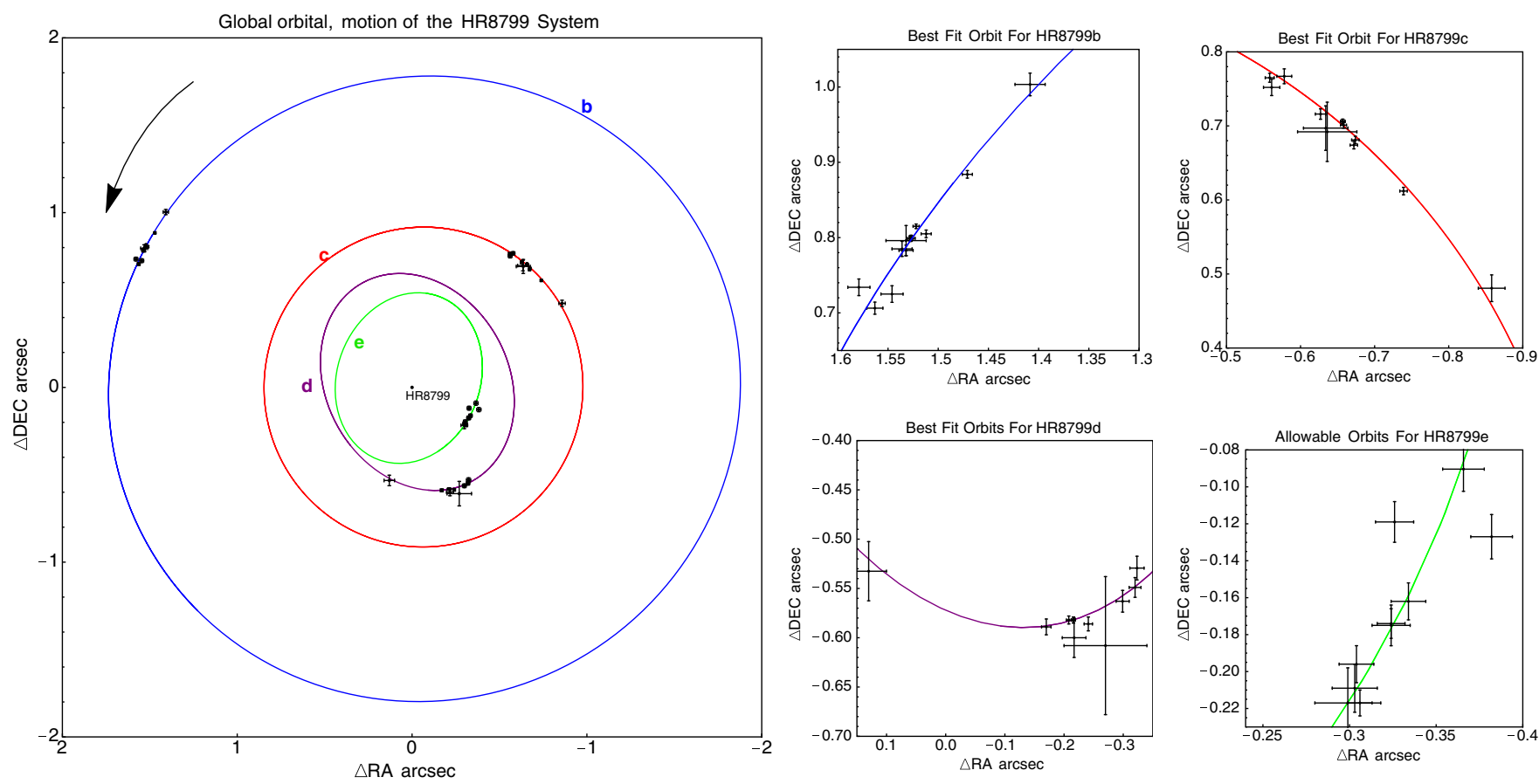

Figure 9. On-sky projection of the best-fit orbits for the four planets orbiting HR 8799. The right panel zooms in on the portions of the orbits that have been observed in the 1998-2012 time span. Note that because HR 8799d seems to be orbiting the host star in a different plane than the other three planets, its on-sky trajectory appears closer to the one of HR 8799e than it actually is. Gray circles denote the Project 1640 epoch, which is the latest epoch considered in our analysis.

Table 2

Astrometric Error Budget

\begin{tabular}{|c|c|c|c|c|}
\hline Planet & $\sigma_{\mathrm{PA}}{ }^{\mathrm{a}}$ & $\sigma_{\mathrm{PS}}^{\mathrm{b}}$ & $\sigma_{\text {Star }}{ }^{c}$ & $\sigma_{\mathrm{KLIP}}{ }^{\mathrm{d}}$ \\
\hline HR 8799b & 0.0064 & $0 ! .0031$ & $0 . ' 0065$ & 0.0039 \\
\hline HR 8799c & 0.'0034 & 0.0017 & 0.0065 & 0.'0039 \\
\hline HR 8799d & $0 ! .0022$ & $0^{\prime \prime} 0011$ & $0 ! .0065$ & 0!.011 \\
\hline HR 8799e & 0!.0014 & $0^{\prime \prime} 00069$ & $0 ! .0065$ & $0^{\prime \prime} 011$ \\
\hline
\end{tabular}

\section{Notes.}

${ }^{\mathrm{a}} \sigma_{\mathrm{PA}}$ : uncertainty on P.A. offset.

${ }^{\mathrm{b}} \sigma_{\mathrm{PS}}$ : uncertainty on plate scale determination.

${ }^{c} \sigma_{\text {Star }}$ : uncertainty on host-star location in the focal plane array.

d $\sigma_{\text {KLIP }}$ : uncertainty stemming from residual errors induced by the KLIP reduction and the forward modeling estimator.

categories: nonlinear least-squares fit of Keplerian elements and dynamical studies. Because of the long orbital periods and the currently limited orbital phase coverage, the parameter landscape explored by nonlinear least-squares methods comprises a multitude of local minima, making it very difficult to unambiguously determine the six Keplerian elements for each planet separately. Recent papers have estimated the most likely orbital architectures assuming either a set inclination for the four planets (Lafrenière et al. 2009; Bergfors et al. 2011; Esposito et al. 2013) or orbits that are coplanar and locked in mean motion resonances ${ }^{18}$ (Soummer et al. 2011a; Currie et al. 2012, 2014). On the other hand, dynamical analysis can constrain the dynamical mass of the planets (upon finding orbital architectures stable over durations at least as long as the estimated stel-

18 Note that Currie et al. $(2012,2014)$ also conducted an orbital motion analysis without any resonant assumption and naturally obtained looser constraints on Keplerian elements than when assuming a Laplace mean motion resonance. lar age) and can predict near-future orbital position (Fabrycky \& Murray-Clay 2010; Reidemeister et al. 2009; Goździewski \& Migaszewski 2009; Esposito et al. 2013; Gozdziewski \& Migaszewski 2013; Marois et al. 2010b). However, dynamical models are generally used in conjunction with strong assumptions regarding coplanarity and mean motion resonances. It was recently shown, under such assumptions, that one could also include planetary migration mechanisms in a dynamical analysis and thus deliver joint information regarding the planets' formation history and masses (Gozdziewski \& Migaszewski 2013). When invoking mean motion resonances to stabilize the system, most authors identified the Laplace 1:2:4:8 resonance as a promising architecture that is compatible with both the available astrometric epochs and masses in the planetary regime $\left(5-10 M_{\text {Jup }}\right)$. The objective of the present paper is to complement both approaches, either a nonlinear least-squares fit or dynamical studies, by answering the following question: "What is the most likely set of Keplerian elements for each planet in the HR 8799 system given the data at hand from 1998 to 2012"'? To do so, we carry out a Bayesian analysis of the published astrometric epochs. Because our approach does not need to resort to any assumptions about the architecture of the system (coplanarity in particular) or the planetary masses, it should provide a good empirical baseline to test published dynamically favorable architectures. We also seek to complement more "data-oriented" methods that either need strong assumptions on the orbital architecture of the system or do not take full advantage of the tools provided by Bayesian inference (Soummer et al. 2011a; Currie et al. 2012, 2014; Lafrenière et al. 2009; Bergfors et al. 2011; Esposito et al. 2013).

Bayesian inference using Markov chain Monte Carlo methods has been extensively used for the detection and characterization of exoplanets using indirect methods: radial velocity (Ford 2005, 

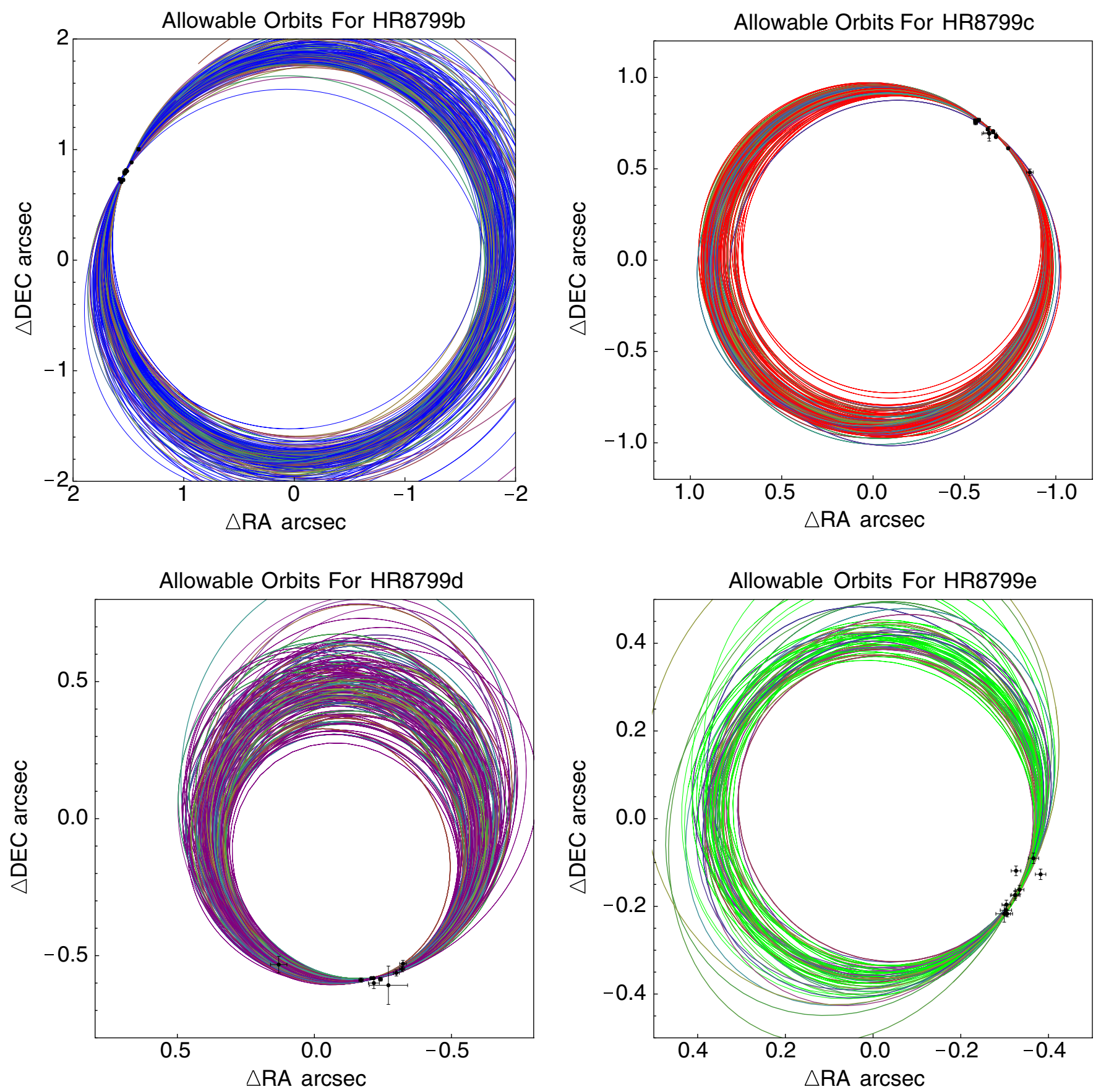

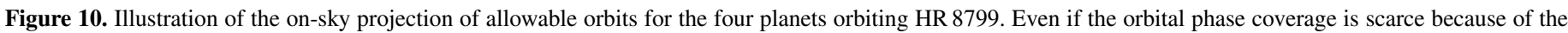
long orbital period of the planets, our Bayesian analysis applied to the 1998-2012 data constrains the orbital architecture of the system.

2006; Gregory 2011; Hou et al. 2012), transits (Eastman et al. 2013), and gravitational microlensing (Skowron et al. 2011). Recently, the direct-imaging community has focused on similar methods to characterize the orbits of Beta Pictoris b (Chauvin et al. 2012) and Fomalhault b (Kalas et al. 2013). We conducted our analysis of the orbital architecture of HR 8799 using two difference MCMC samplers: the Metropolis Hastings algorithm discussed in Ford (2006), Chauvin et al. (2012), Kalas et al. (2013) and the Affine Invariant Sampler described in ForemanMackey et al. (2013). Both approaches did yield almost identical posterior distributions for the six orbital elements of each planet. We direct the reader to the aforementioned publications for a detailed description of our methodology, and below we only describe the broad lines of our Bayesian analysis:
1. We use all of the published epochs summarized in Esposito et al. (2013), augmented by our P1640 points.

2. For each planet, we seek to constrain the six orbital elements: period $P$, eccentricity $e$, inclination $i$, longitude of ascending node $\Omega$, argument of periastron $\omega$, and epoch at periastron $t_{P}$. We consider the following state vector $\mathbf{x}=\left(\log (P), e, \cos i, \omega+\Omega, \omega-\Omega, t_{p}\right)$.

3. Case of the Metropolis-Hasting Sampler: our prior distributions of periods for each planet are uniform, centered around the "circular face on period" ( $P_{\text {cfo }}$, not to be confused with the commonly eccentric orbit $P_{\text {ceo }}$ ) derived from the discovery epochs and covering more than a full decade around that value, $P_{\text {prior }} \in\left[P_{\text {cfo }} / 4,4 P_{\text {cfo }}\right]$. Because high eccentricities have been ruled out (Marois et al. 2010b), 






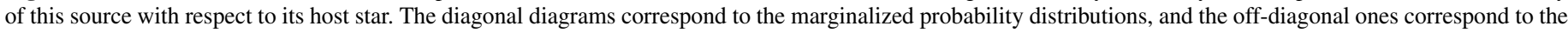
correlation between various parameters.

we initialize our chains according to a uniform distribution spanning $[0,0.8]$. We assume a uniform prior distribution for $\cos i \in[-1,1], \omega \in[0,2 \pi], \Omega \in[0,2 \pi]$, and $t_{p} \in\left[P_{\text {cfo }}-4 P_{\text {cfo }}, P_{\text {cfo }}+4 P_{\text {cfo }}\right]$. We improve convergence by using the transformation $\mathbf{u}(\mathbf{x})$ described in the Appendix of Chauvin et al. (2012) and use for $t_{0}$ the origin of mean anomalies (Ford 2006), the epoch with the largest number of contemporaneous observations (usually the epoch at which confirmation of physical association was unambiguously established). Because the orbital phase coverage is much smaller than in the case of Beta Pictoris $b$ and more similar to Fomalhault b, we further improve convergence by using a parallel tempering ladder (Gregory 2005; Kalas et al. 2013).

4. Affine Invariant Sampler: We follow the recommendation of Foreman-Mackey et al. (2013), and we first initialized our walkers in a small ball centered on the most likely orbital elements derived using the Metropolis-Hastings Sampler. We then take advantage of the enhanced computational speed of the affine invariant sampler and explore a variety of walker initialization points within the range discussed above. We verify that the better-behaved chains in terms of acceptance rate, autocorrelation, and overall chi-squared are indeed the ones corresponding to the most likely orbital 







correlation between various parameters.

elements estimated using the Metropolis-Hasting sampler. We use these latter chains for inference.

5. Both the small orbital phase coverage and the high starto-planet mass ratios do not allow us to take advantage of our inference chains to carry out the class dynamical mass estimates described in Dupuy et al. (2009), Konopacky et al. (2010), and Crepp et al. (2012). Because we cannot reach this level of precision, we do not marginalize over the distance to HR 8799 (assumed to be $36.4 \mathrm{pc}$ ) or the host stellar mass (assumed to be $1.51 M_{\text {Sun }}$ ).
Our results for each planet are shown in Figures 11-14. The elements of the "most likely orbit" and $1 \sigma$ confidence intervals indicated are summarized in Table 3 , and the orbits associated with the lowest $\chi^{2}$ value are displayed in Figure 9. Finally, the ensemble of allowable orbits, located in the $1 \sigma$ confidence intervals, is shown in Figure 10; this illustrates the degeneracies associated with fitting Keplerian orbital elements using astrometric data spanning only a small portion of orbital phase. Nevertheless, even in the presence of such degeneracies, the marginalized probability density function in Figures 11-14 can help us to significantly constrain the architecture of the 


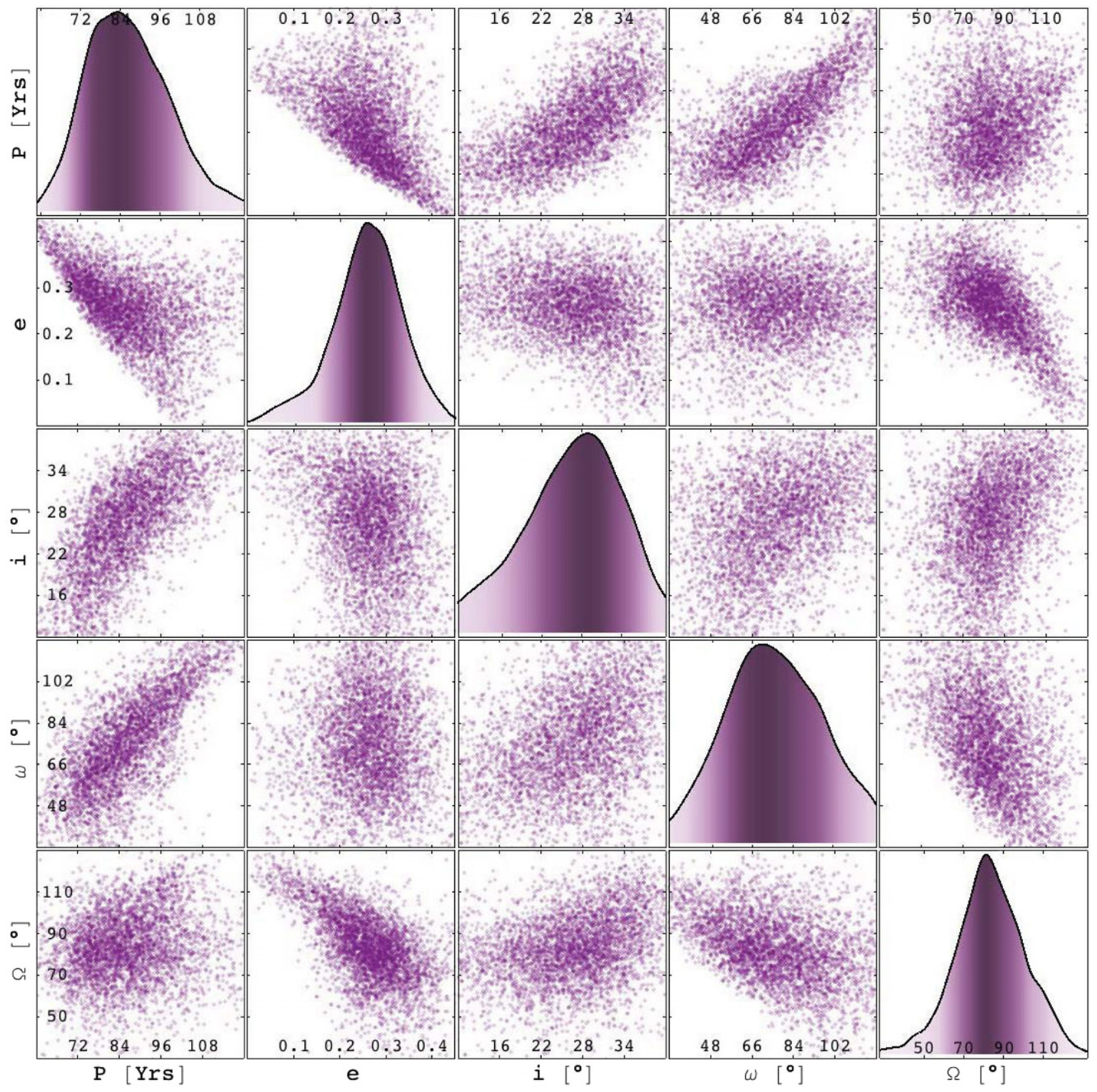

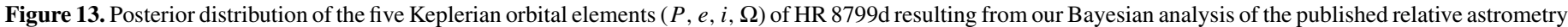

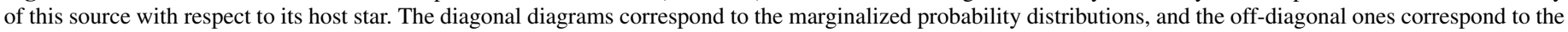
correlation between various parameters.

HR 8799 system. We discuss these aspects in Section 4. In a future paper we will test the dynamical stability of this ensemble of allowable orbits or order to obtain a more precise understanding of the orbital architectures of the system and of the planet's dynamical masses.

However, before delving into our interpretation of Figures 11-14, we remind the reader of the caveats associated with our Bayesian analysis of the astrometric history of this system. Markov chain Monte Carlo methods are known to be very sensitive to systematic underestimated biases (e.g., not captured by published error bars) in the various astrometric measurements. The astrometric data supporting the analysis of Chauvin et al. (2012) and Kalas et al. (2013) was extremely homogenous because it was based on two instruments at most, and all of the imaging data reduction had been conducted by a single team. In contrast, the astrometric history underlying our analysis is heterogeneous and comprises estimates stemming from at least six observatories and a variety of data analysis approaches. For instance, should small discrepancies between absolute north calibrations, beyond the reported error bars, occur between observatories, then the posterior distributions in Figures 11-14 will be biased. This could, for instance, be the source of the minor differences between our results and the confidence intervals reported in Currie et al. (2012, 2014). Although our results for HR $8799 \mathrm{bcd}$ are generally in good agreement with Currie et al. (2012, 2014), some of our confidence intervals do not overlap. The presence of biased observations in the astrometric history of HR 8799 combined with the sensitivity of MCMC approaches to such unaccounted uncertainties could be the sources of these discrepancies. Alternatively, it could be that 




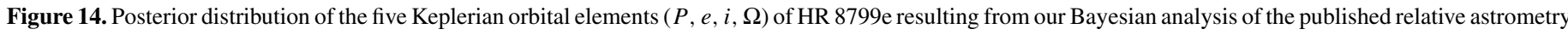

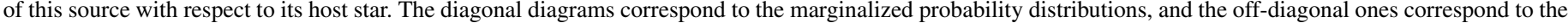
correlation between various parameters.

the approach in Currie et al. $(2012,2014)$ does not sufficiently explore the $\chi^{2}$ landscape. Homogeneous observations with newgeneration extreme adaptive optics instruments, which possess superior astrometric accuracy, will largely resolve this issue over the upcoming decade. Note, however, that the systematics between observatories (and epochs if necessary) could be included in the MCMC state vector, just as mean radial velocities are included in observations of the reflex motion of exoplanetary host stars along the line of sight (Ford 2006). Should this approach be carried out, it would then yield systematics-free (and thus more reliable) confidence intervals for the architecture of a directly imaged exoplanetary system. Although this would be an extremely interesting academic exercise, including potential systematics in our analysis is beyond the scope of the present paper.

\section{DISCUSSION}

\subsection{Coplanarity}

In order to test coplanarity, we then folded the inclinations and longitudes of ascending notes in Figure 11-14 to compute the direction of the vector orthogonal to the orbital plane of each realization of the orbits of HR879bcde. We then used as 


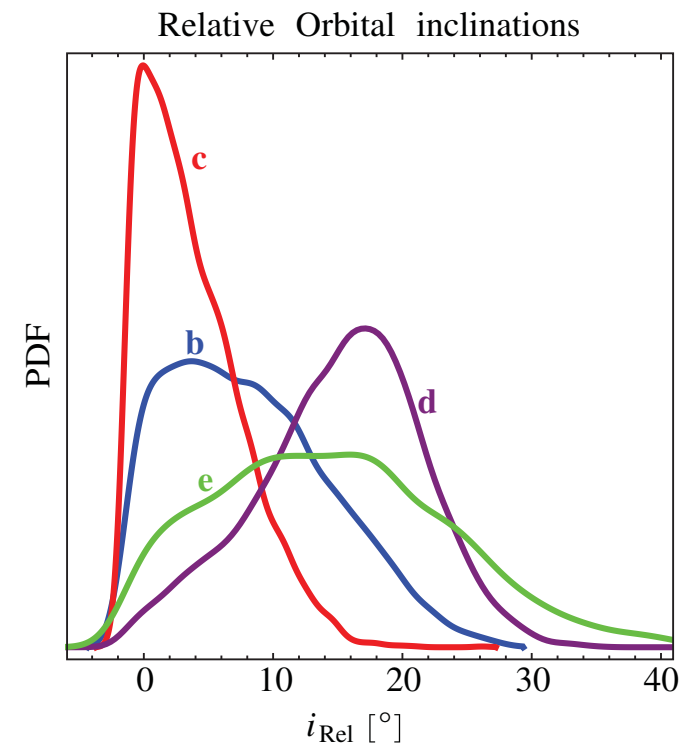

Figure 15. Coplanarity test for the four planets in the HR 8799 system. This figure shows the relative inclinations of the orbital planes with respect to the most likely inclination (whereas the inclinations in Figures 11-14 are with respect to the line of sight). While this figure does not unambiguously rule out coplanarity, it suggests that HR 8799bc most likely orbits in the same plane, and HR 8799d's orbit is out of the plane. More data is needed to constrain the plane of the orbit of HR 8799e.

Table 3

Most Likely Keplerian Elements for the Planets in the HR 8799 System and Confidence Intervals

\begin{tabular}{lcccc}
\hline \hline & HR 8799b & HR 8799c & HR 8799d & HR 8799e \\
\hline$\left.P_{\chi_{\min }^{2}}(\mathrm{Yr})\right]$ & 525.3 & 174.5 & 87.4 & 58.9 \\
$P, 1 \sigma$ & {$[479.5,574.9]$} & {$[164.1,184.9]$} & {$[74.5,99.8]$} & {$[45.1,70.3]$} \\
\hline$e_{\chi_{\min }^{2}}$ & 0.056 & 0.086 & 0.26 & 0.14 \\
$e, 1 \sigma$ & {$[0.018,0.092]$} & {$[0.042,0.12]$} & {$[0.18,0.33]$} & {$[0.045,0.21]$} \\
\hline$i_{\chi_{\min }^{2}}\left[{ }^{\circ}\right]$ & 17.2 & 10.5 & 26.3 & 25.5 \\
$i, 1 \sigma$ & {$[8.5,25.9]$} & {$[5.1,16.0]$} & {$[18.2,34.2]$} & {$[13.3,37.5]$} \\
\hline$\omega \chi_{\min }^{2}\left[{ }^{\circ}\right]$ & 134.1 & 123.9 & 76.4 & 160.7 \\
\hline, $1 \sigma$ & {$[102.8,164.9]$} & {$[100.1,147.5]$} & {$[53.1,99.9]$} & {$[111.7,206.0]$} \\
\hline$\Omega \chi_{\min }^{2}\left[{ }^{\circ}\right]$ & 69.4 & 81.4 & 82.9 & 89.0 \\
$\Omega, 1 \sigma$ & {$[46.5,91.1]$} & {$[60.2,101.1]$} & {$[66.8,100.4]$} & {$[58.3,128.3]$} \\
\hline
\end{tabular}

a reference direction the vector orthogonal to the orbital plane of the most likely orbit of HR 8799c, and Figure 15 shows the relative inclinations of each planet with respect to this reference. While this figure does not unambiguously rule out coplanarity, it suggests that HR 8799bc most likely orbits in the same plane, while HR 8799d's orbit is out of the plane. More data is needed to constrain the plane of the orbit of HR 8799e. This noncoplanar orbital architecture has not been included in recently published dynamical analyses. As a final sanity check, we further test the robustness of our analysis regarding the orbits of HR 8799d. A direct inspection of Figure 9 hints that the out-of-plane best fit for HR 8799d might stem from a biased astrometry in the 1998 HST-NICMOS epoch (Soummer et al. 2011a) because the large temporal lever arm is responsible for strong constraints on the orbit. We tested the robustness of our results to this bias by removing the HST-NICMOS point from the astrometric history. This test did yield posterior distributions of orbital elements similar to Figure 13 (albeit with larger uncertainties in the period when removing the 1998 epoch). We can thus rule out this

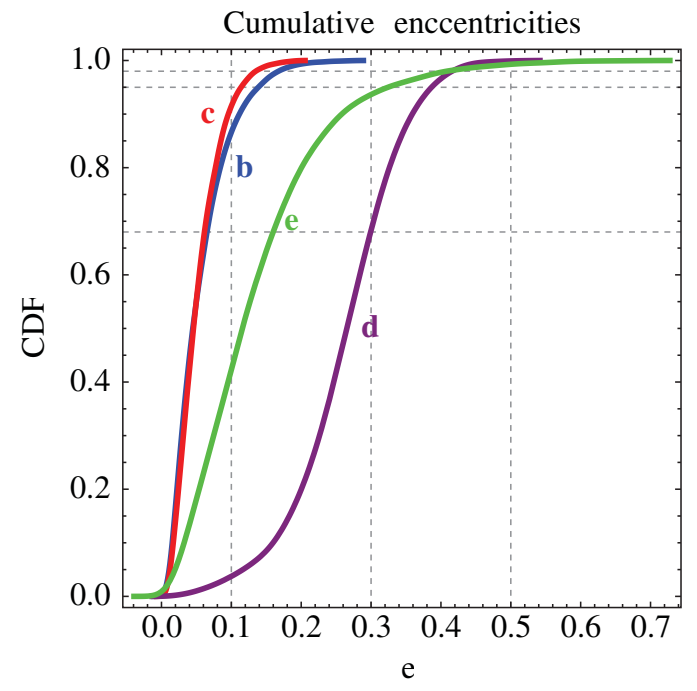

Figure 16. Cumulative distributions of the eccentricities of the four planets orbiting HR 8799. The dashed horizontal lines, from bottom to top, correspond to the $68 \%, 95 \%$, and $98 \%$ confidence levels. Our analysis of the current astrometric history confirms the published eccentricity hierarchy in other works, with HR 8799d being the most eccentric planet and HR $8799 \mathrm{bc}$ featuring almost circular orbits $(e<0.1$ with $68 \%$ confidence). More orbital coverage will be necessary to firmly establish the eccentricity of HR 8799e.

scenario and conclude that, in the absence of other unidentified pathological cases, the orbit of HR $8799 \mathrm{~d}$ is misaligned by $\sim 15^{\circ}-20^{\circ}$ compared to the roughly coplanar HR 8799 bce orbits.

\subsection{Eccentricities}

All four planets appear to orbit HR 8799 with low eccentricities, which has been predicted using dynamical arguments. Indeed, a circularizing mechanism ought to have occurred in the youth of this system in order for it to have lasted a few tens of million years (Fabrycky \& Murray-Clay 2010). Figure 16 shows the cumulative distribution of eccentricities in the HR 8799 system, with horizontal lines denoting the $68 \%, 95 \%$, and $98 \%$ confidence levels. The overall eccentricity distribution is consistent with other studies, such as by Soummer et al. (2011a), Reidemeister et al. (2009), and Gozdziewski \& Migaszewski (2013), which identified HR 8799d as the most eccentric planet: the $1 \sigma$ upper limits for $e_{b}, e_{c}, e_{e}$ are respectively $0.07,0.06,0.12$, and the same upper limit of HR 8799d is 0.3 . Thus, in spite of the noncoplanarity discussed above, our analysis of the astrometric history of this system hints that HR 8799d seems to have a special role in the eccentric hierarchy. The fact that HR $8799 \mathrm{~d}$ orbits at a larger eccentricity and a likely off-plane inclination might be the fingerprints of dynamical interactions during the formation of this planetary system.

\subsection{Mean Motion Resonances}

Mean motion resonances play a crucial role in stabilizing the HR 8799 system, as first pointed out by Fabrycky \& MurrayClay (2010). While resonances are not strictly limited to integer period ratios, we identify on Figures 17 the lowest-order ratios that are compatible with our analysis of the astrometric history of the system. We find:

1. $b$-c resonance our analysis seems to favor a period ratio between $5 b: 2 c$ and $3 b: 1 c$. Interestingly, the $2 b: 1 c$ resonance 

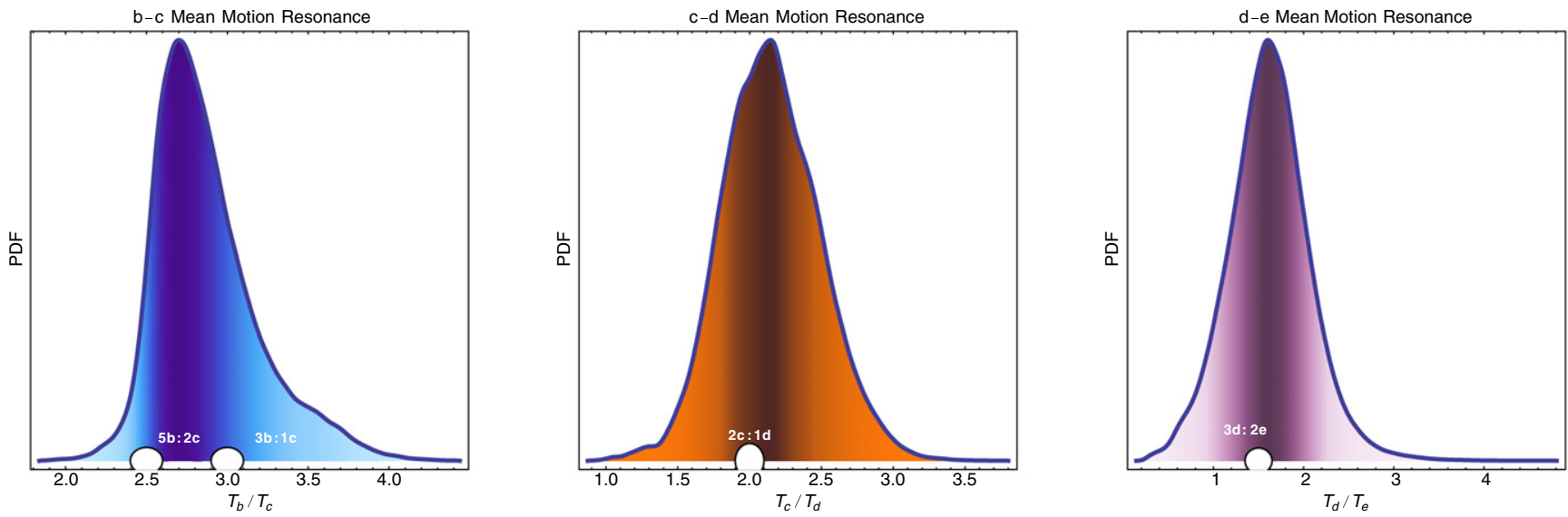

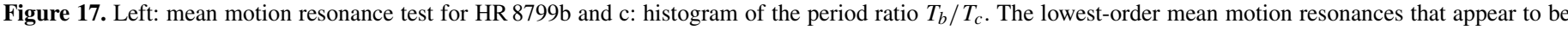

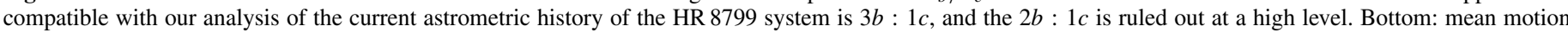

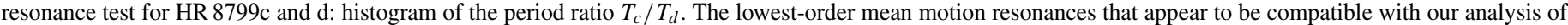

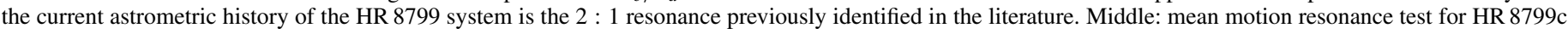

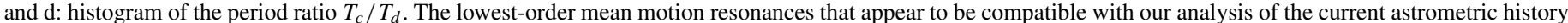

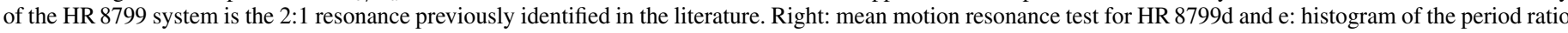

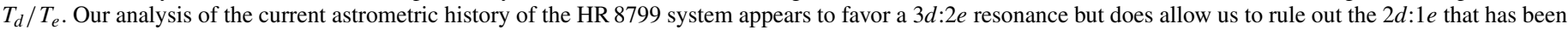
previously suggested in the literature.


Figure 18. Left: zeroth order dynamical stability test of HR $8799 \mathrm{~d}$ and e. Percentage of the orbits allowed by our analysis of the current astrometric history of the HR 8799 system that preclude a "close encounter" with the next orbital period of HR $8799 \mathrm{~d}$ as a function of each planet's masses. We indicate in red the boundary of the domain where more than $68 \%$ of the combinations of our allowable orbits do not feature a "close encounter. While this is rough test from a dynamical standpoint, the absence of any orbit pairs without close encounters for masses larger than $12 M_{\text {Jup }}$ indicates that the HR 8799d and e dynamical masses most likely lie below this threshold. Center: zeroth order dynamical stability test of HR $8799 \mathrm{c}$ and d. Percentage of the orbits allowed by our analysis of the current astrometric history of the HR 8799 system that preclude a "close encounter" with the next orbital period of HR 8799c as a function of each planet's masses. Right: zeroth order dynamical stability test of HR 8799b and c. Percentage of the orbits allowed by our analysis of the current astrometric history of the HR 8799 system that preclude a "close encounter" with the next orbital period of HR $8799 \mathrm{~b}$ as a function of each planet's masses.

that had been identified as a promising candidate by dynamical studies assuming coplanarity between the two objects can be ruled out by our analysis.

2. $c$ - $d$ resonance our analysis seems to favor period ratios commensurate with a $2 \mathrm{c}: 1 \mathrm{~d}$ resonance.

3. $d$-e resonance our analysis yields a period ratio histogram centered around a 3:2e resonance but is also compatible with a 2d:1e. Further astrometric monitoring of HR 8799e is required to disentangle these scenarios.

Thus, even if HR 8799d most likely does not orbit in the same plane as HR 8799c and e, the period ratios involving these planets do not rule out a 1e:2c:4d Laplace resonance. However, they do favor a $3 \mathrm{~d}: 2 \mathrm{e}$ resonance for the inner pair of planets. The period ratio for the two outer planets favored by our analysis does not suggest a Laplace resonance but is consistent with Currie et al. (2012, 2014). Because resonances are a strong mechanism to stabilize multiple planetary systems, the confidence intervals derived in the present paper can serve as a good first guess to study secular dynamical interaction in the HR 8799 system. Future $N$-body analyses of this system should thus be carried out to complement our work and further constrain the period ratios discussed above.

\subsection{Likelihood of Close Encounter}

The tight bounds on the dynamical mass of HR 8799 bcde can potentially be estimated via $N$-body simulations over the lifetime of the system for the ensembles of allowable Keplerian elements derived by our analysis. However, this exercise is beyond the scope of the present paper, and we will devote a future communication to this matter (A. Veicht et al. 2014, in preparation). Here we nevertheless illustrate how such an exercise could be used to bound the dynamical mass of planets 
in this system based on our results in Figures 11-14. Our method consists of calculating the fraction of allowable orbits that pass the "close-encounter test": following the arguments of Chatterjee et al. (2008), we define as a close encounter an epoch for which the two planets are within four mutual Hill radii. For each object, we draw a set of 1000 Keplerian elements according to the posterior distributions of our Bayesian analysis. We then calculate the position of each planet in a three-dimensional frame centered on the star over the next $10^{3} \mathrm{yr}$ (about twice the period of HR 8799b). For each neighboring pair of objects (b-c, $\mathrm{c}-\mathrm{d}$, and $\mathrm{d}-\mathrm{e}$ ), we compute the distance between the $\sim 10^{6}$ pairs of outer-inner orbits, vary the mass of the objects, and estimate the percentage of orbit combinations that do not feature a close encounter as defined above.

Our results are illustrated in Figure 18, where we display the likelihood of orbits compatible with the astrometric history of HR 8799 without close encounters, assuming the posterior distributions discussed in Section 3. The top right of the two right-most panels shows that none of the orbits estimated by our analysis of the astrometric history of HR 8799 yields dynamically stable orbits for masses $>60 M_{\mathrm{Jup}}$. In contrast, the bottom left indicates the region for which more than $68 \%$ of the combinations of our allowable orbits do not feature a close encounter (indicated by a red dashed line).

Note that this boundary is arbitrary and only shown for illustration purposes. Indeed, only one stable orbital configuration $\left(10^{-4} \%\right.$ in our case) suffices to yield an orbital architecture without a close encounter. Indeed, no rigorous dynamical mass bounds can be firmly established for HR 8799 b-c using Figure 18 because the large scatter in orbital elements resulting from our orbital motion analysis can lead to artificially low masses. However, this figure provides an indication of how dynamical considerations will be able to further constrain orbital parameters and masses. Note, moreover, that our close-encounter criterion is extremely loose when it comes to constraining dynamical masses: the distance boundary defined Chatterjee et al. (2008) is designed to rule out orbital near approaches that will result in ejections in the next $10^{5} \mathrm{yr}$. Clearly, more subtle dynamical interactions can occur on scales larger than four mutual Hill radii. Moreover, the duration of our calculation is conservatively limited to twice the period of HR $8799 \mathrm{~b}$ (we assume that at such short timescales the secular perturbations of orbital elements are negligible), which is clearly not sufficient to explore all of the possible geometries.

In contrast, we find that all of the orbit pairs underlying the left-most panel of Figure 18 feature a close encounter if the masses of HR $8799 \mathrm{~d}-\mathrm{e}$ are above $12 M_{\mathrm{Jup}}$. While the $68 \%$ boundary might be artificially low because of orbital element scatter, the lack of stable orbits above $12 M_{\text {Jup }}$ indicates that it is very unlikely that orbits matching the astrometric history of this system will yield dynamically stable configurations unless the masses of at least HR 8799de are below $13 M_{\text {Jup }}$ (unless there remain unidentified pathological biases in our Bayesian analysis). Note that here we adopt $m<13 M_{\text {Jup }}$ as a "planetary mass" for the sake of argument, in spite of the boundary in the planet/brown dwarf classification being truly tied to formation history and thus still an active area of investigation. This is, to our knowledge, the most "assumption-free" constraint on the dynamical masses in the HR 8799 planetary system because our analysis only relies on the very weak assumptions regarding the uncorrelated prior distributions of Keplerian elements for each planet. This approach complements the current literature that focused on identifying dynamically stable configurations while assuming coplanarity or circular orbits (Fabrycky \& MurrayClay 2010; Gozdziewski \& Migaszewski 2013; Esposito et al. 2013; Reidemeister et al. 2009). In a future communication, we will substitute this loose close-encounter criterion with full $\mathrm{N}$ body simulations and thus merge both approaches in order to shed further light on the orbital architectures of this planetary system (A. Veicht et al. 2014, in preparation).

\section{CONCLUSION}

In this paper we have presented a new astrometric perspective on the four substellar objects orbiting HR 8799. We relied on the same Project 1640 observations that lead to a parent publication focused on their spectral characterization (Oppenheimer et al. 2013). In that paper we demonstrated how the combination of state-of-the-art coronagraphs and adaptive optics systems with an integral field spectrograph could provide tremendous insights into the atmospheric diversity of such purported planets. Here we first focused on the intricacies associated with astrometric estimation using such a complex system. In particular, we introduced two new algorithms, one that retrieves the stellar focal plane position (even when masked by a coronagraphic stop), and another that yields precise astrometry and spectrophotometry of faint point sources even when they are initially buried in the speckle noise. This latter algorithm was built upon a recent publication by Soummer et al. (2012) and is now becoming a standard tool in the field of high-contrast imaging. The principal component analysis underlying our KLIP algorithm can moreover be furthered to capture the true three-dimensional stochastic nature of the speckles in Integral Field Spectrograph data, as demonstrated in a future publication reporting a novel method also developed by the P1640 team (Fergus et al. 2013). We hope that our detailed discussions regarding the intricacies of incoherent speckle suppression in high-contrast Integral Field Spectrographs, and the tenuous tasks of estimating stellar location in the presence of a coronagraphs, will facilitate upcoming large-scale surveys.

The second part of our paper was devoted to the interpretation of the published astrometric history of HR 8799, augmented with our Project 1640 epoch. In order to complement the various interpretations currently in the literature, we conducted a Bayesian analysis based on the Markov Chain Monte Carlo method, using the methods described in Ford (2006), Chauvin et al. (2012), and Kalas et al. (2013). Under the caveats associated with the sensitivity of such a method to unaccounted astrometric biases, we were able to determine an ensemble of likely Keplerian orbits for HR 8799 bcde without any prior assumptions on the overall configuration of the system. We then discussed the implications of our results in terms of orbital architecture, which are summarized below.

1. The four planets appear to be coplanar in the broad sense of our outer solar system. However, HR 8799d orbits slightly outside of the plane of HR 8799 bce, with a misalignment in relative inclination of $\sim 15^{\circ}$. More data is necessary to unambiguously rule out coplanarity.

2. It is particularly interesting to note that planet d appears to have both a different inclination and eccentricity. If confirmed in the future with additional orbital data, this result would be particularly interesting to help understand the history of the system dynamic, where some event might have pumped both eccentricity and inclination into a system otherwise mostly circularized and coplanar.

3. The majority of recent publications discussing the orbital architecture and dynamic stability of the HR 8799 system 
assumed strict coplanarity for the four planets. Our results raise questions about the validity of this assumption and as a consequence yield an updated eccentricities hierarchy and most-likely period ratios different than previously thought.

4. Based on the set of most-likely orbits established by our analysis of the astrometric history and a loose dynamical survival argument based on geometric close encounters, we have established a very high likelihood of masses below $13 M_{\text {Jup }}$ for HR 8799de and illustrated how future dynamical analyses will further constrain dynamical masses in the entire system.

In an upcoming publication, we will propagate our ensemble of likely orbits through $\mathrm{N}$-body simulations in order to further constrain this likely subset of configurations to the few architectures that are favored by the data. This effort will eventually provide robust dynamical mass estimates and, when combined with our low-resolution spectroscopic observations, will provide a critical piece to the current puzzle associated with the formation history of this system.

\section{REFERENCES}

Amara, A., \& Quanz, S. P. 2012, MNRAS, 427, 948

Baraffe, I., Chabrier, G., \& Barman, T. 2010, RPPh, 73, 016901

Barman, T. S., Macintosh, B., Konopacky, Q. M., \& Marois, C. 2011a, ApJ, 733, 65

Barman, T. S., Macintosh, B., Konopacky, Q. M., \& Marois, C. 2011b, ApJL, 735, L39

Beichman, C. A., Krist, J., Trauger, J. T., et al. 2010, PASP, 122, 162

Bergfors, C., Brandner, W., Janson, M., Köhler, R., \& Henning, T. 2011, A\&A, 528, A134

Beuzit, J.-L., Feldt, M., Dohlen, K., et al. 2008, Proc. SPIE, 7014, 701418

Bouchez, A. H., Dekany, R. G., Angione, J. R., et al. 2008, Proc. SPIE, 7015,70150

Bowler, B. P., Liu, M. C., Dupuy, T. J., \& Cushing, M. C. 2010, ApJ, 723, 850

Brandt, T. D., McElwain, M. W., Janson, M., et al. 2014, in Proc. SPIE, 9148, 49

Brandt, T. D., McElwain, M. W., Turner, E. L., et al. 2013, ApJ, 764, 183

Brandt, T. D., McElwain, M. W., Turner, E. L., et al. 2014, ApJ, 794, 159

Chatterjee, S., Ford, E. B., Matsumura, S., \& Rasio, F. A. 2008, ApJ, 686, 580

Chauvin, G., Lagrange, A.-M., Beust, H., et al. 2012, A\&A, 542, A41

Chauvin, G., Lagrange, A.-M., Dumas, C., et al. 2005, A\&A, 438, L25

Crepp, J. R., \& Johnson, J. A. 2011, ApJ, 733, 126

Crepp, J. R., Johnson, J. A., Fischer, D. A., et al. 2012, ApJ, 751, 97

Crepp, J. R., Pueyo, L., Brenner, D., et al. 2011, ApJ, 729, 132

Currie, T., Burrows, A., Girard, J. H., et al. 2014, ApJ, 795, 133

Currie, T., Burrows, A., Itoh, Y., et al. 2011, ApJ, 729, 128

Currie, T., Fukagawa, M., Thalmann, C., Matsumura, S., \& Plavchan, P. 2012, ApJL, 755, L34

Dekany, R., Roberts, J., Burruss, R., et al. 2013, ApJ, 776, 130

Digby, A. P., Hinkley, S., Oppenheimer, B. R., et al. 2006, ApJ, 650, 484

Dupuy, T. J., Liu, M. C., \& Bowler, B. P. 2009, ApJ, 706, 328

Eastman, J., Gaudi, B. S., \& Agol, E. 2013, PASP, 125, 83

Esposito, S., Mesa, D., Skemer, A., et al. 2013, A\&A, 549, A52

Fabrycky, D. C., \& Murray-Clay, R. A. 2010, ApJ, 710, 1408

Fergus, R., Hogg, D. W., Brenner, D., \& Oppenheimer, B. R. 2014, ApJ, 794, 161

Ford, E. B. 2005, AJ, 129, 1706

Ford, E. B. 2006, ApJ, 642, 505

Foreman-Mackey, D., Hogg, D. W., Lang, D., \& Goodman, J. 2013, PASP, 125,306

Galicher, R., Marois, C., Macintosh, B., Barman, T., \& Konopacky, Q. 2011, ApJL, 739, L41

Goździewski, K., \& Migaszewski, C. 2009, MNRAS, 397, L16

Gozdziewski, K., \& Migaszewski, C. 2014, MNRAS, 440, 3140

Gregory, P. C. 2005, Bayesian Logical Data Analysis for the Physical Sciences: A Comparative Approach with 'Mathematica' Support (Cambridge: Cambridge Univ. Press)
Gregory, P. C. 2011, MNRAS, 410, 94

Guizar-Sicairos, M., Thurman, S. T., \& Fienup, J. R. 2008, OptL, 33, 156

Hartkopf, W. I., Mason, B. D., \& Worley, C. E. 2001, AJ, 122, 3472

Hinkley, S., Carpenter, J. M., Ireland, M. J., \& Kraus, A. L. 2011a, ApJL, 730, L21

Hinkley, S., Oppenheimer, B. R., Zimmerman, N., et al. 2011b, PASP, 123,74

Hinkley, S., Pueyo, L., Faherty, J. K., et al. 2013, ApJ, 779, 153

Hinz, P. M., Rodigas, T. J., Kenworthy, M. A., et al. 2010, ApJ, 716, 417

Hou, F., Goodman, J., Hogg, D. W., Weare, J., \& Schwab, C. 2012, ApJ, 745,198

Ireland, M. J., Kraus, A., Martinache, F., Law, N., \& Hillenbrand, L. A. 2011, ApJ, 726, 113

Janson, M., Bergfors, C., Goto, M., Brandner, W., \& Lafrenière, D. 2010, ApJL, 710, L35

Janson, M., Carson, J., Lafreniere, D., et al. 2012, ApJ, 747, 116

Kalas, P., Graham, J. R., Chiang, E., et al. 2008, Sci, 322, 1345

Kalas, P., Graham, J. R., Fitzgerald, M. P., \& Clampin, M. 2013, ApJ, 775,56

Konopacky, Q. M., Barman, T. S., Macintosh, B. A., \& Marois, C. 2013, Sci, 339, 1398

Konopacky, Q. M., Ghez, A. M., Barman, T. S., et al. 2010, ApJ, 711, 1087

Lafrenière, D., Jayawardhana, R., \& van Kerkwijk, M. H. 2008, ApJL, 689, L153

Lafrenière, D., Marois, C., Doyon, R., \& Barman, T. 2009, ApJL, 694, L148

Lafrenière, D., Marois, C., Doyon, R., Nadeau, D., \& Artigau, É. 2007, ApJ, 660,770

Lagrange, A., Bonnefoy, M., Chauvin, G., et al. 2010, Sci, 329, 57

Macintosh, B. A., Graham, J. R., Palmer, D. W., et al. 2008, Proc. SPIE, 7015, 701518

Madhusudhan, N., Burrows, A., \& Currie, T. 2011, ApJ, 737, 34

Marley, M. S., Saumon, D., Cushing, M., et al. 2012, ApJ, 754, 135

Marois, C., Correau, C., Galicher, K., et al. 2014, Proc. SPIE, 9148, 91480

Marois, C., Lafrenière, D., Doyon, R., Macintosh, B., \& Nadeau, D. 2006a, ApJ, 641,556

Marois, C., Lafrenière, D., Macintosh, B., \& Doyon, R. 2006b, ApJ, 647,612

Marois, C., Lafrenière, D., Macintosh, B., \& Doyon, R. 2008a, ApJ, 673, 647

Marois, C., Macintosh, B., Barman, T., et al. 2008b, Sci, 322, 1348

Marois, C., Macintosh, B., \& Véran, J. 2010a, Proc. SPIE, 7736

Marois, C., Zuckerman, B., Konopacky, Q. M., Macintosh, B., \& Barman, T. 2010b, Natur, 468, 1080

McBride, J., Graham, J. R., Macintosh, B., et al. 2011, PASP, 123, 692

Milli, J., Mouillet, D., Lagrange, A.-M., et al. 2012, A\&A, 545, A111

Oppenheimer, B. R., Baranec, C., Beichman, C., et al. 2013, ApJ, 768, 24

Oppenheimer, B. R., \& Hinkley, S. 2009, ARA\&A, 47, 253

Pueyo, L., Crepp, J. R., Vasisht, G., et al. 2012, ApJS, 199, 6

Pueyo, L., Wallace, K., Troy, M., et al. 2010, Proc., SPIE, 7736

Quanz, S. P., Meyer, M. R., Kenworthy, M. A., et al. 2010, ApJL, 722, L49

Reidemeister, M., Krivov, A. V., Schmidt, T. O. B., et al. 2009, A\&A, 503,247

Savransky, D., Thomas, S. J., Poyneer, L. A., \& Macintosh, B. A. 2013, Appl. Opt., 52, 3394

Serabyn, E., Mawet, D., \& Burruss, R. 2010, Natur, 464, 1018

Sivaramakrishnan, A., \& Oppenheimer, B. R. 2006, ApJ, 647, 620

Skemer, A. J., Hinz, P. M., Esposito, S., et al. 2012, ApJ, 753, 14

Skowron, J., Udalski, A., Gould, A., et al. 2011, ApJ, 738, 87

Soummer, R. 2005, ApJL, 618, L161

Soummer, R., Brendan Hagan, J., Pueyo, L., et al. 2011a, ApJ, 741, 55

Soummer, R., Pueyo, L., \& Larkin, J. 2012, ApJL, 755, L28

Soummer, R., Pueyo, L., Sivaramakrishnan, A., \& Vanderbei, R. J. 2007, OExpr, 15,15935

Soummer, R., Sivaramakrishnan, A., Pueyo, L., Macintosh, B., \& Oppenheimer, B. R. 2011b, ApJ, 729, 144

Sparks, W. B., \& Ford, H. C. 2002, ApJ, 578, 543

Spiegel, D. S., \& Burrows, A. 2012, ApJ, 745, 174

Vasisht, G., Cady, E., Ligon, E. R., et al. 2014, Proc. SPIE, 9148, 914822

Veras, D., Crepp, J. R., \& Ford, E. B. 2009, ApJ, 696, 1600

Wallace, J. K., Burruss, R., Pueyo, L., et al. 2009, Proc. SPIE, 7440, 74400 S

Yelda, S., Lu, J. R., Ghez, A. M., et al. 2010, ApJ, 725, 331

Zimmerman, N., Brenner, D., Oppenheimer, B. R., et al. 2011, PASP, 123,746

Zimmerman, N., Oppenheimer, B. R., Hinkley, S., et al. 2010, ApJ, 709, 733 